Dislocation subgrain structures and modeling the plastic hardening of metallic single crystals

This article has been downloaded from IOPscience. Please scroll down to see the full text article.

2010 Modelling Simul. Mater. Sci. Eng. 18055001

(http://iopscience.iop.org/0965-0393/18/5/055001)

View the table of contents for this issue, or go to the journal homepage for more

Download details:

IP Address: 131.215.220.165

The article was downloaded on 12/07/2010 at 18:28

Please note that terms and conditions apply. 


\title{
Dislocation subgrain structures and modeling the plastic hardening of metallic single crystals
}

\author{
B L Hansen ${ }^{1,2}$, C A Bronkhorst ${ }^{1}$ and M Ortiz ${ }^{2}$ \\ 1 Theoretical Division, Los Alamos National Laboratory, NM 87545, USA \\ 2 Division of Engineering and Applied Science, California Institute of Technology, Pasadena, \\ CA 91125, USA \\ E-mail: bhansen@lanl.gov
}

Received 23 August 2009, in final form 26 March 2010

Published 29 April 2010

Online at stacks.iop.org/MSMSE/18/055001

\begin{abstract}
A single crystal plasticity theory for insertion into finite element simulation is formulated using sequential laminates to model subgrain dislocation structures. It is known that local models do not adequately account for latent hardening, as latent hardening is not only a material property, but a nonlocal property (e.g. grain size and shape). The addition of the nonlocal energy from the formation of subgrain structure dislocation walls and the boundary layer misfits provide both latent and self-hardening of a crystal slip. Latent hardening occurs as the formation of new dislocation walls limits motion of new mobile dislocations, thus hardening future slip systems. Self-hardening is accomplished by an evolution of the subgrain structure length scale. The substructure length scale is computed by minimizing the nonlocal energy. The minimization of the nonlocal energy is a competition between the dislocation wall energy and the boundary layer energies. The nonlocal terms are also directly minimized within the subgrain model as they affect deformation response. The geometrical relationship between the dislocation walls and slip planes affecting the dislocation mean free path is taken into account, giving a firstorder approximation to shape effects. A coplanar slip model is developed due to requirements while modeling the subgrain structure. This subgrain structure plasticity model is noteworthy as all material parameters are experimentally determined rather than fit. The model also has an inherit path dependence due to the formation of the subgrain structures. Validation is accomplished by comparison with single crystal tension test results.
\end{abstract}

(Some figures in this article are in colour only in the electronic version) 


\section{Introduction}

Crystal plasticity has been a topic of interest since the deformation of metals was investigated. Original research into the field of crystal plasticity led to the discovery of dislocations, which is the primary mechanism of plasticity. Further work has been concerned with the interaction of these dislocations concerning hardening. As dislocations move along certain crystallographic slip planes during deformation, the bulk motions of dislocations are accumulated into a quantity known as slip. These slip systems harden by interaction of dislocations, either within the same plane (self-hardening) or by dislocation motion on a different slip plane (latent hardening). Many of the mechanisms that give rise to the attributes exhibited in latent hardening experiments are under investigation (Havner 2005).

Previous work investigating microstructures and plasticity has uncovered that if a single crystal deformed by plastic slip has latent hardening and is allowed to form an arbitrarily fine subgrain dislocation structure without any energy penalty for the accumulation of dislocations, then each slip system will appear macroscopically to harden independently (Conti and Ortiz 2005). This occurs because the microstructure forms in such a way as to limit interaction between slip systems. This observation is in conflict with experimental data demonstrating that two active slip systems exhibit latent hardening (Franciosi and Zaoui 1982). As research also indicates that latent hardening is not a material property, but rather depends on the crystal grain in which the material deforms (e.g. grain shape and size), it is realized that the observed latent hardening is a property of interactions due to the grain properties. This work attempts to add hardening due to subgrain dislocation structure interactions into a constitutive crystal plasticity code for use as a material model in finite element calculations.

Previous studies (Ortiz and Repetto 1999, Conti and Ortiz 2005) have provided evidence that with strong latent hardening the energy of single slip is an energy well and is always less than multiple slip in energy. Under this assumption of strong latent hardening, the only way for a material to activate multiple slip systems is to divide into spatial volumes with different single slip systems. According to previous studies (Ortiz and Repetto 1999, Ortiz et al 2000, Aubrey and Ortiz 2003) microstructures of single slip regions will be introduced when the energy of the microstructure is less than the energy of single slip (or purely elastic deformation). This work deviates from previous studies when the dislocation structures are formulated by introducing coplanar slip laminates and in the calculation of the laminate length scale from the nonlocal energy terms. The coplanar slip model is used due to the lack of dislocation wall formation between two active coplanar slip systems. If the coplanar model is not used, the hardening is not appropriately calculated.

Figures 1 and 2 display the formation of typical subgrain dislocation structures in two different copper specimens taken using a transmission electron microscope. The images are courtesy of Cerreta (2008). Figure 1 is a cell structure of dislocation entanglement frequently seen during initial plastic deformation. The walls between the cells are formed by the accumulation of random dislocations that cause a very low angle misorientation. These are frequently referred to as incidental dislocation boundaries (IDBs). Upon further deformation, the cell walls tend to elongate and increase in misorientation angle to form parallel dislocation walls similar to a laminate structure as seen in figure 2. The formation of the elongated walls is associated with a strain difference across the walls. These are called geometrically necessary boundaries (GNBs). For a full review of subgrain dislocation laminate structures the reader is referred to the works of Hughes and Hansen (1997), Hughes et al (1997) and Hansen et al (2001). These works include an analysis of the subgrain microstructures on which this model is based as well as scaling laws for the structures. 


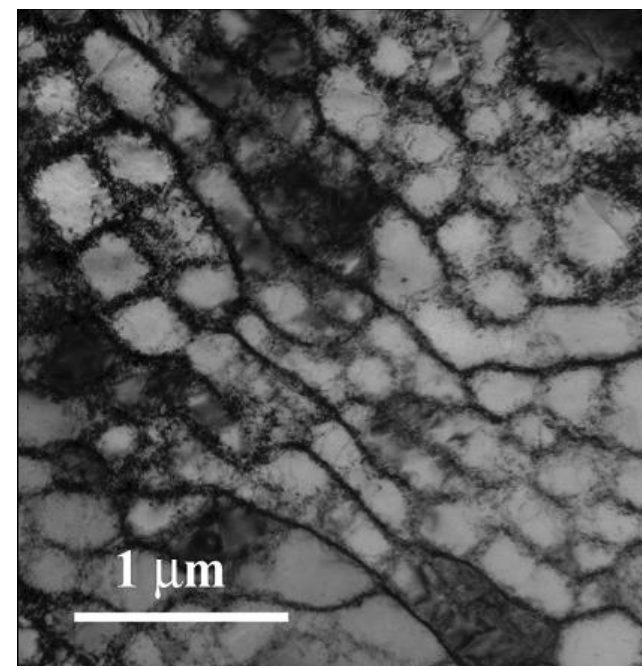

Figure 1. Classic subgrain cell structure in high-purity copper (reproduced with permission (Cerreta 2008)).

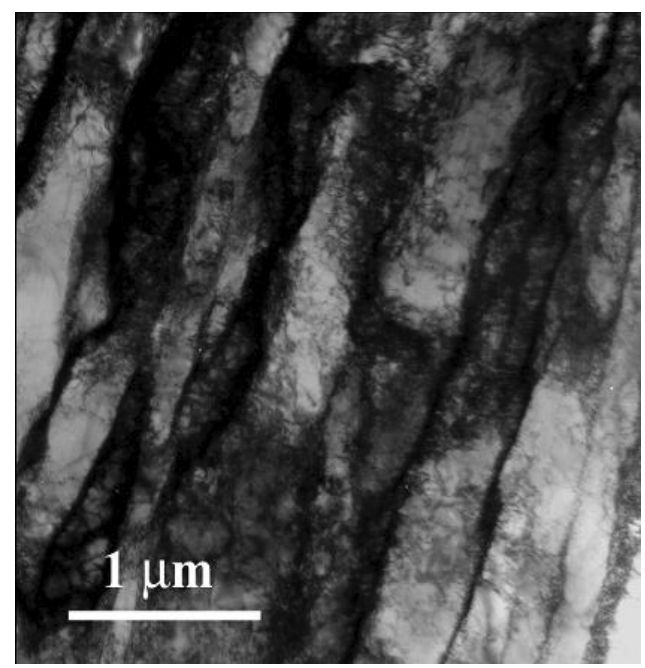

Figure 2. Laminate subgrain structure formed by elongation of cell walls in copper (reproduced with permission (Cerreta 2008)).

Steeds (1966) provides a study of single crystal copper dislocation structures as a function of strain. The work of Ortiz and Repetto (1999) contains a comparison between the laminate microstructure model used in this work and many experimentally observed subgrain structures. Dmitrieva et al (2009) recently found that the formation of banded microscopic lattice rotation patterns in sheared copper single crystals could be produced by a lamination model.

The work of Ortiz et al (2000) followed in which the ideas of Ortiz and Repetto (1999) were implemented into a finite deformation constitutive model that explicitly resolved subgrain 
dislocation laminate structures. It demonstrated the possibility of capturing grain size effects on deformation and contains the first implementation of nonlocal energy into the laminar microstructures. Comparisons are made between the direct simulation of subgrain dislocation microstructures to obtain size effects and the nature of size effects obtained by the strain gradient method.

The previous work was built upon by Aubrey and Ortiz (2003), in which the model was streamlined for easier energy calculations and infinite latent hardening. Aubrey and Ortiz compared the scaling of the formed microstructures with the scaling law of Hughes et al (1997). Much of this current work follows directly from the ideas of Aubrey and Ortiz, but varies considerably in the implementation of those ideas. This work also compares more directly with experimental measurements.

At this point, the theory of laminate dislocation microstructures has diverged onto several distinct paths. The work of Aubrey et al (2003) considered the relaxation of martensitic materials using a subgrain laminate microstructure with branching and pruning operations. The work of Conti et al (2007) considered the application of minimization principles directly rather than explicitly resolving the microstructure, such that calculations can determine stress states and the laminate dislocation microstructures can be resolved after calculations are complete. This is being further explored by Gurses and Ortiz (2009). The work of Conti and Ortiz (2005) considered linearized kinematics and the formation of microstructures. Conti and Theil (2005) considered single slip finite deformation microstructures in which elastic structures are permitted.

An understanding of traditional plasticity theories is useful for understanding this work, as many sound principles from those works are used. A selection of models includes Cuitino and Ortiz (1993) and Bronkhorst et al (2007). It should be emphasized that these are local models which contain latent hardening in multiple slip, but do not attempt to model subgrain dislocation structure or nonlocal energy.

In this work, the model introduced by Ortiz and Repetto (1999), Ortiz et al (2000) and investigated by Aubrey and Ortiz (2003) is further developed. Differences and extensions of the current methodology are highlighted. The new developments in this work include the evolution of the subgrain microstructure lengths to treat hardening behavior, a method that accounts for the unique features of coplanar slip systems, the effects of grain shape and dislocation cell wall normal orientation on the mean free path of dislocations, direct incorporation of the nonlocal energy in the minimization of deformation energy (rather than as a perturbation), corrections to the boundary layer energy formulation and an investigation of the subgrain laminate wall normal implementation.

In section 2, the general constitutive framework of a single crystal incorporating both elastic and plastic deformation is presented. Coplanar slip is developed separately after single slip. The general framework is then applied to laminate microstructures which include nonlocal energy terms in section 3. The nonlocal energy terms are derived after the laminate microstructures as this gives the presentation a more concise flow, although the reader needs to accept the nonlocal terms before they are developed. This order prevents the presentation of the model initially without nonlocal terms and then a second time with the nonlocal terms included. The coplanar structures are again discussed afterward. The equilibration of the microstructure for new deformation states is described. Significant changes have been added to the nonlocal energy and equilibration. Finally, the model is validated by simulating experimental tests of copper single crystals (section 4). Comparisons are made with variations in single crystal stress response due to crystal orientation, grain size and grain shape. The subgrain structures are also compared with traditional single crystal plasticity theories. Conclusions are drawn in section 5 . 


\section{Material model}

The model is concerned with determining the reaction of a crystalline material occupying a volume $\Omega$ when a deformation mapping $y[x]: \Omega \rightarrow R^{3}$ is applied. The deformation gradient is defined by

$$
\boldsymbol{F}[\boldsymbol{x}] \equiv \nabla y[\boldsymbol{x}],
$$

where $\nabla$ is the gradient operator. The brackets [.] are used to represent a functional dependence in this work, whereas (.) are used to show order of operations or to distinguish superscripts from exponents.

In anticipation of applying the model at a material integration point within a continuum scale application any dependence on the position variable $\boldsymbol{x}$ will be dropped. Thus, all equations apply for a single given $\boldsymbol{x}$, i.e. $\boldsymbol{F}[\boldsymbol{x}] \rightarrow \boldsymbol{F}$. The value $\boldsymbol{F}$ will be referenced as the 'global' or 'macroscopic' deformation. The behavior at the material point $\boldsymbol{x}$ is being simulated.

Indices will be used frequently in notation. To keep the meaning of indices clear, they will always be placed in identical positions. The upper right will be used for modifying quantities, such as differentiating between plastic and elastic values; the lower left for discretization or iteration; and the lower right for other enumeration, such as over slip systems or laminate structures.

$$
\text { discretization } X_{\text {enumeration }}^{\text {modifier }}
$$

\subsection{Constitutive framework}

First, a Helmholtz free energy density $\phi[\boldsymbol{F}, \boldsymbol{q}]$ per undeformed unit of volume which depends upon the deformation gradient $\boldsymbol{F}$ and a set of internal state variables $\boldsymbol{q}$ is considered. No temperature or strain rate dependence is taken into account in this work. When no viscosity is present, Coleman's relations give the first Piola-Kirchoff stress tensor, $\boldsymbol{P}$, as

$$
\boldsymbol{P}=\frac{\partial \phi[\boldsymbol{F}, \boldsymbol{q}]}{\partial \boldsymbol{F}} .
$$

The material tangent is defined by

$$
\mathcal{C} \equiv \frac{\partial \boldsymbol{P}}{\partial \boldsymbol{F}}
$$

The general material formulation for the tangents can be found in Ortiz and Stainier (1999).

Following Rice (1975), an inelastic potential, $\psi$, is also introduced along with the corresponding thermodynamic force, $Q$, conjugate to the internal variables $\boldsymbol{q}$ :

$$
\begin{gathered}
\boldsymbol{Q}=-\frac{\partial \phi[\boldsymbol{F}, \boldsymbol{q}]}{\partial \boldsymbol{q}}, \\
\dot{\boldsymbol{q}}=\frac{\partial \psi[\boldsymbol{Q}, \boldsymbol{q}]}{\partial \boldsymbol{Q}},
\end{gathered}
$$

where a "., indicates time derivative. All these relations are subject to material frame indifference (see Ortiz et al (2000) and Ortiz and Stainier (1999) for consequences).

\subsection{Single crystal model}

The proceeding framework is now applied to a single crystal. The full deformation of a material point is composed of multiple deformation gradients, an elastic, followed by multiple plastic. 
A multiplicative decomposition of the deformation gradient proposed by Lee (1969) is adopted:

$$
\boldsymbol{F}=\boldsymbol{F}^{\mathrm{e}} \boldsymbol{F}^{\mathrm{p}}
$$

where $\boldsymbol{F}^{\mathrm{e}}$ is an elastic deformation which distorts the crystalline lattice and $\boldsymbol{F}^{\mathrm{p}}$ is the total plastic deformation caused by dislocation motion. The plastic deformation is assumed to leave the crystal lattice undistorted, at least at the macroscopic continuum level.

For metallic crystals, it is also reasonable to assume that the energy can be split into an elastic portion, $W^{\mathrm{e}}$, and a dislocation-based plastic portion, $W^{\mathrm{p}}$.

$$
\phi=W^{\mathrm{e}}\left[\boldsymbol{F}^{\mathrm{e}}\right]+W^{\mathrm{p}}\left[\boldsymbol{F}^{\mathrm{p}}, \gamma_{\alpha}\right]
$$

where $\gamma_{\alpha}$ is the plastic shear strain on slip system $\alpha$. Application of (2.2) gives

$$
\boldsymbol{P}=\frac{\partial W^{\mathrm{e}}\left[\boldsymbol{F}^{\mathrm{e}}\right]}{\partial \boldsymbol{F}^{\mathrm{e}}}\left(\boldsymbol{F}^{\mathrm{p}}\right)^{-\mathrm{T}}
$$

The stress tensor

$$
\boldsymbol{P}^{\mathrm{e}} \equiv \boldsymbol{P}\left(\boldsymbol{F}^{\mathrm{p}}\right)^{\mathrm{T}}
$$

is defined for later use.

Following the formulation of Ortiz and Repetto (1999) the internal variables are $\boldsymbol{q}=$ $\left(\boldsymbol{F}^{\mathrm{p}}, \gamma_{\alpha}\right)$ and the conjugate forces are $\boldsymbol{Q}=(\boldsymbol{S},-g)$. Computing the forces from (2.4) yields

$$
\boldsymbol{S} \equiv-\frac{\partial \phi}{\partial \boldsymbol{F}^{\mathrm{p}}}=\left(\boldsymbol{F}^{\mathrm{e}}\right)^{\mathrm{T}} \frac{\partial W^{\mathrm{e}}}{\partial \boldsymbol{F}^{\mathrm{e}}}\left(\boldsymbol{F}^{\mathrm{p}}\right)^{-\mathrm{T}}
$$

which is related to the symmetric second Piola-Kirchoff stress tensor, and

$$
g \equiv \frac{\partial \phi}{\partial \gamma_{\alpha}} .
$$

It follows from (2.5) that

$$
\begin{aligned}
& \dot{\boldsymbol{F}}^{\mathrm{p}}=\frac{\partial \psi}{\partial \boldsymbol{S}}, \\
& \dot{\gamma}_{\alpha}=-\frac{\partial \psi}{\partial g} .
\end{aligned}
$$

Note that $\dot{\gamma}_{\alpha}$ is the rate of slip strain on slip system $\alpha$.

2.2.1. Elastic deformation model. For the elastic portion of the deformation, a Saint VenantKirchoff material is used. It is defined by the following equations. Starting with the GreenLagrange strain tensor

$$
\boldsymbol{E}^{\mathrm{e}}=\frac{1}{2}\left(\left(\boldsymbol{F}^{\mathrm{e}}\right)^{\mathrm{T}} \boldsymbol{F}^{\mathrm{e}}-\boldsymbol{I}\right),
$$

where $\boldsymbol{I}$ is the identity tensor, the second Piola-Kirchoff stress tensor follows by assuming a linear relationship with the crystal's elasticity constants, $\mathcal{C}^{\text {mat }}$,

$$
\boldsymbol{K}^{\mathrm{e}}=\mathcal{C}^{\text {mat }} \cdot \boldsymbol{E}^{\mathrm{e}}
$$

An assumption of linearity is accurate for the elastic portion of deformation for metallic materials (Cuitino and Ortiz 1993). Higher order moduli are available in Teodosiu (1982). The first Piola-Kirchoff stress tensor for an elastic deformation follows as

$$
\boldsymbol{P}^{\mathrm{e}}=\boldsymbol{F}^{\mathrm{e}} \boldsymbol{K}^{\mathrm{e}} \text {. }
$$

One needs to note that $\boldsymbol{P}^{\mathrm{e}}$ relates to the overall $\boldsymbol{P}$ by use of (2.9). 
Table 1. FCC slip systems.

\begin{tabular}{|c|c|c|c|c|c|c|}
\hline System & A2 & A3 & A6 & B2 & B4 & B5 \\
\hline$s * \sqrt{2}$ & $\pm\left[\begin{array}{lll}0 & \overline{1} & 1\end{array}\right]$ & $\pm\left[\begin{array}{lll}1 & 0 & 1\end{array}\right]$ & $\pm\left[\begin{array}{lll}1 & 1 & 0\end{array}\right]$ & $\pm\left[\begin{array}{lll}0 & \overline{1} & 1\end{array}\right]$ & $\pm\left[\begin{array}{lll}1 & 0 & 1\end{array}\right]$ & $\pm\left[\begin{array}{lll}\overline{1} & 1 & 0\end{array}\right]$ \\
\hline$m * \sqrt{3}$ & $\left(\begin{array}{lll}1 & 1 & 1\end{array}\right)$ & $\left(\begin{array}{lll}1 & 1 & 1\end{array}\right)$ & $\left(\begin{array}{lll}1 & 1 & 1\end{array}\right)$ & $\left(\begin{array}{lll}1 & 1 & 1\end{array}\right)$ & $\left(\begin{array}{lll}1 & 1 & 1\end{array}\right)$ & $\left(\begin{array}{lll}1 & 1 & 1\end{array}\right)$ \\
\hline System & $\mathrm{C} 1$ & $\mathrm{C} 3$ & $\mathrm{C} 5$ & D1 & D4 & D6 \\
\hline$s * \sqrt{2}$ & $\pm\left[\begin{array}{lll}0 & 1 & 1\end{array}\right]$ & $\pm\left[\begin{array}{lll}1 & 0 & 1\end{array}\right]$ & $\pm\left[\begin{array}{lll}\overline{1} & 1 & 0\end{array}\right]$ & $\pm\left[\begin{array}{lll}0 & 1 & 1\end{array}\right]$ & $\pm\left[\begin{array}{lll}1 & 0 & 1\end{array}\right]$ & $\pm\left[\begin{array}{lll}1 & 1 & 0\end{array}\right]$ \\
\hline$m * \sqrt{3}$ & $\left(\begin{array}{lll}\overline{1} & \overline{1} & 1\end{array}\right)$ & $\left(\begin{array}{lll}\overline{1} & \overline{1} & 1\end{array}\right)$ & 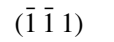 & $(1 \overline{1} 1)$ & $\left(\begin{array}{lll}1 & 1 & 1\end{array}\right)$ & $\left(\begin{array}{lll}1 & \overline{1} & 1\end{array}\right)$ \\
\hline
\end{tabular}

The elastic energy density is computed as

$$
W^{\mathrm{e}}=\frac{1}{2} \boldsymbol{K}^{\mathrm{e}} \cdot \boldsymbol{E}^{\mathrm{e}} .
$$

The material tangent for elastic deformation is given by indicial notation for ease as follows:

$$
\mathcal{C}_{i J k L}^{\mathrm{e}}=\frac{\partial \boldsymbol{P}^{\mathrm{e}}}{\partial \boldsymbol{F}^{\mathrm{e}}}=\delta_{i k} K_{J L}^{\mathrm{e}}+2 F_{i M}^{\mathrm{e}} F_{k N}^{\mathrm{e}} \mathcal{C}_{M J N L}^{\mathrm{mat}}
$$

2.2.2. Plastic deformation model. The shear stress acting on slip system $\alpha$ is resolved geometrically as

$$
\tau_{\alpha}=\boldsymbol{P}\left(\boldsymbol{F}^{\mathrm{p}}\right)^{\mathrm{T}} \cdot\left(\boldsymbol{F}^{\mathrm{e}} \boldsymbol{s}_{\alpha} \otimes \boldsymbol{m}_{\alpha}\right)=\boldsymbol{S}\left(\boldsymbol{F}^{\mathrm{p}}\right)^{\mathrm{T}} \cdot\left(\boldsymbol{s}_{\alpha} \otimes \boldsymbol{m}_{\alpha}\right),
$$

where the $\cdot$ operator between two higher order tensors is defined as $A \cdot M=A_{. . k l} M_{k l . .}$, using Einsteinian notation to indicate the indices $k$ and $l$ are summed over; $\boldsymbol{m}_{\alpha}$ is the slip plane normal and $s_{\alpha}$ is the slip direction of system $\alpha$. Again following Rice (1975), the flow rule is given a potential structure by assuming the potential of each slip system can be summed:

$$
\psi\left[\boldsymbol{S}, g ; \boldsymbol{F}^{\mathrm{p}}, \gamma\right]=\sum_{\alpha=1}^{N} \psi_{\alpha}\left[\boldsymbol{S}, g_{\alpha} ; \boldsymbol{F}^{\mathrm{p}}, \gamma_{\alpha}\right] .
$$

An application of Schmidt's rule, $\psi_{\alpha}=\psi_{\alpha}\left[\tau_{\alpha}-g_{\alpha}\right]$, demonstrates that $g$ is the critical resolved shear stress, $\tau_{\alpha}^{\mathrm{c}}$, or stress at which slip system $\alpha$ activates.

The flow rule for plastic deformation is obtained by applying (2.12) and (2.13):

$$
\begin{aligned}
& \dot{\boldsymbol{F}}^{\mathrm{p}}=\left(\sum_{\alpha=1}^{N} \frac{\partial \psi_{\alpha}}{\partial \tau_{\alpha}} \boldsymbol{s}_{\alpha} \otimes \boldsymbol{m}_{\alpha}\right) \boldsymbol{F}^{\mathrm{p}}, \\
& \dot{\gamma}_{\alpha}=-\frac{\partial \psi_{\alpha}}{\partial g_{\alpha}}=\frac{\partial \psi_{\alpha}}{\partial \tau_{\alpha}} .
\end{aligned}
$$

It is apparent that the flow rule for plastic deformation is that introduced by Rice (1971), i.e.

$$
\dot{\boldsymbol{F}}^{\mathrm{p}}=\left(\sum_{\alpha=1}^{N} \dot{\gamma}_{\alpha} \boldsymbol{s}_{\alpha} \otimes \boldsymbol{m}_{\alpha}\right) \boldsymbol{F}^{\mathrm{p}},
$$

where $\alpha$ is a summation over all crystallographic slip systems.

In this work, slip is regarded as irreversible and thus,

$$
\dot{\gamma}_{\alpha} \geqslant 0 \text {. }
$$

For reference the 12 slip systems of FCC crystals are given in table 1 . Since the systems are considered irreversible, there are 24 systems for FCC crystals in this model. 
Assuming rate-independent slip and minimizing the deformation energy, it is shown in Ortiz and Repetto (1999) that the slip rates and stresses can be restricted to the Kuhn-Tucker form (Rockafellar 1970) as

$$
\tau_{\alpha}-g_{\alpha} \leqslant 0, \quad \dot{\gamma}_{\alpha} \geqslant 0, \quad\left(\tau_{\alpha}-g_{\alpha}\right) \dot{\gamma}_{\alpha}=0 .
$$

These conditions provide an optimization technique and all the traditional constraints for rateindependent plasticity. Namely, that the material remains elastic, with plastic deformation occurring only when critical resolved shear stresses are reached, and that slip is irreversible. A strain rate dependent model could be developed by including a strain rate dependent evolution of plastic deformation (see e.g. Bronkhorst et al (2007) or Cuitino and Ortiz (1993)).

It is also noted from (2.11), (2.7) and $g_{\alpha}=\tau_{\alpha}^{\mathrm{c}}$ during slip that

$$
W^{\mathrm{p}}=\sum_{\alpha=1}^{N} \int_{t=0}^{t^{*}} \tau_{\alpha}^{\mathrm{c}}[t] \dot{\gamma}_{\alpha}[t] \mathrm{d} t
$$

The material tangent when a single slip system is active is

$\mathcal{C}_{i J k L}^{\alpha}=C_{i M k N}^{\mathrm{e}}\left(F^{\mathrm{p}}\right)_{J M}^{-1}\left(F^{\mathrm{p}}\right)_{L N}^{-1}-C_{i J \alpha}^{\text {mixed }} C_{k L \alpha}^{\text {mixed }} \frac{1}{\partial\left(\tau_{\alpha}-\tau_{\alpha}^{c}\right) / \partial \gamma_{\alpha}}$,

where

$$
\mathcal{C}_{i j \alpha}^{\text {mixed }}=\mathcal{C}_{i m a b}^{\mathrm{e}} F_{a q}^{\mathrm{e}} s_{q}^{\alpha}\left(F^{\mathrm{p}}\right)_{m j}^{-\mathrm{T}} m_{b}^{\alpha}+P_{i m}^{\mathrm{e}}\left(F^{\mathrm{p}}\right)_{j q}^{-1} s_{q}^{\alpha} m_{m}^{\alpha}
$$

and with the assumptions $\frac{\partial \gamma_{\alpha}}{\partial\left(\tau_{\alpha}-\tau_{\alpha}^{\mathrm{c}}\right)}=1 / \frac{\partial\left(\tau_{\alpha}-\tau_{\alpha}^{\mathrm{c}}\right)}{\partial \gamma_{\alpha}}$ and that $\boldsymbol{s}_{\alpha} \cdot \boldsymbol{m}_{\alpha}=0$. The equation for $\frac{\partial\left(\tau_{\alpha}-\tau_{\alpha}^{\mathrm{c}}\right)}{\partial \gamma_{\alpha}}$ is given in the general form for two coplanar slip systems in (2.38).

\subsection{Numerical implementation}

It is easiest to define all tensors in the crystal frame. No equation refers to the lab frame in this section. Obviously, the results must be rotated back to the lab frame, and inputs from the lab frame must be rotated into the crystal frame when this material model is implemented for multiple crystal orientations. Working in the crystal frame simplifies many equations as crystal material properties are easier to express in the crystal frame, such as $s, \boldsymbol{m}$ and $\mathcal{C}$. Thus, there are only two frames of reference, the undeformed crystal and the deformed crystal.

2.3.1. Incremental deformation steps. Time discretization is used by enforcing the boundary conditions at discrete time steps: $\left(t_{0}, t_{1}, \ldots, t_{n}, t_{n+1}, \ldots\right)$. The subscript $n$ and $n+1$ will be used to distinguish values at different time steps.

2.3.2. Elastic. The only required input to the elastic model is the elastic deformation gradient, ${ }_{n+1} \boldsymbol{F}^{\mathrm{e}}$. Elastic deformation does not have a path dependence and as there is no time dependence in the elastic model, the time discretization does not need any discussion.

2.3.3. Crystal single slip. A system which allows only single slip is adopted. The energy for each possible slip system is determined and the minimum energy system is selected, thus determining the active slip system. Note that once a slip system is activated, it will be the only slip system active in that spatial portion of material for all future time steps unless further dislocation microstructure forms. As will be introduced in the next section, the material can escape the single slip restriction only by forming microstructures of multiple single slip regions. This convention is due to the work of Ortiz and Repetto (1999) and Conti and Ortiz (2005) in 
which it was shown that while using a slip model involving discrete crystal slip systems with a strong latent hardening matrix, local single slip provides energy wells in the energy of deformation. Thus with this model, the lowest energy available to accommodate a deformation is always a microstructure of single slip volumes.

Assuming a given active slip system, the single slip model takes four inputs: the total deformation at $t_{n+1},{ }_{n+1} \boldsymbol{F}$; the previous time step's plastic deformation, ${ }_{n} \boldsymbol{F}^{\mathrm{p}}$; the total accumulated slip strain at $t_{n},{ }_{n} \gamma_{\alpha}$; and the current mean free path length of the dislocations, ${ }_{n+1} h_{\alpha}$. The mean free paths and how they are obtained are discussed in section 3.3.1. The total energy density, ${ }_{n+1} W$, stress state, ${ }_{n+1} \boldsymbol{P}$, and updated state variables, ${ }_{n+1} \boldsymbol{F}^{\mathrm{p}}$ and ${ }_{n+1} \gamma_{\alpha}$, need to be computed. The subscript $\alpha$ is maintained to denote that these equations are for a single slip system.

Starting with the change in slip strain on slip system $\alpha$ :

$$
\Delta \gamma_{\alpha}={ }_{n+1} \gamma_{\alpha}-{ }_{n} \gamma_{\alpha},
$$

then from an exponential mapping of equation (2.23)

$$
{ }_{n+1} \boldsymbol{F}^{\mathrm{p}}=\left(\boldsymbol{I}+\sum_{\alpha=1}^{N} \Delta \gamma_{\alpha} \boldsymbol{s}_{\alpha} \otimes \boldsymbol{m}_{\alpha}\right){ }_{n} \boldsymbol{F}^{\mathrm{p}} .
$$

where the sum over $\alpha$ could be reduced to only the single active slip system.

The magnitude of ${ }_{n+1} \gamma_{\alpha}$ is solved by iteration using (2.30) to solve for ${ }_{n+1} \boldsymbol{F}^{\mathrm{p}}$ and (2.6) to solve for ${ }_{n+1} \boldsymbol{F}^{\mathrm{e}}$. The resolved shear stress is computed by passing ${ }_{n+1} \boldsymbol{F}^{\mathrm{e}}$ to the elastic model to obtain ${ }_{n+1} \boldsymbol{P}$ and using equation (2.19). The value of ${ }_{n+1} \gamma_{\alpha}$ can be found by using an iterative method such as the Newton-Raphson until the Kuhn-Tucker conditions (2.25) are satisfied, i.e. the resolved shear stress equals the critical resolved shear stress or in the simplest case, only an elastic reaction occurs.

It should be noted that these values are strictly incremental, yielding a path-dependent nature to the evolution of the material.

The total discretized energy density is defined as

$$
{ }_{n+1} W={ }_{n+1} W^{\mathrm{e}}+{ }_{n+1} W^{\mathrm{p}},
$$

which is introduced to replace $\phi$ in order to keep the symbols uniform for the discretized variables. Also,

$$
{ }_{n+1} W^{\mathrm{p}}=\left({ }_{n+1} \tau_{\alpha}^{\mathrm{c}}\right)\left({ }_{n+1} \gamma_{\alpha}\right),
$$

from (2.26) since only one slip system is active. As derived by Aubrey and Ortiz (2003), the dislocation wall energy has the effect of increasing the critical resolved shear stress as

$$
{ }_{n+1} \tau_{\alpha}^{\mathrm{c}}=\tau_{\mathrm{o}}^{\mathrm{c}}+\frac{T}{b_{n+1} h_{\alpha}},
$$

where $T$ is the dislocation line tension (assumed constant in this work) and $b$ is the Burgers vector. The time integral from (2.26) is dropped since the only change to $\tau_{\alpha}^{\mathrm{c}}$ is from (2.33) and although the mean free path, ${ }_{n+1} h_{\alpha}$, may change, it is assumed that all slip occurs at the same mean free path. This is discussed more in section 3.

Given the value of ${ }_{n+1} \tau_{\alpha}^{\mathrm{c}}$, the energy can be computed from (2.31) (2.17) and (2.32), and the stress from (2.9) and (2.16).

2.3.4. Crystal coplanar slip. It will be shown when laminate dislocation structures are introduced that coplanar slip (referring to slip in different crystallographic directions, but on the same crystallographic slip plane; i.e. $m_{1}=m_{2}, s_{1} \neq s_{2}$ ) requires additional treatment 
to get the appropriate hardening. This is due to the fact that two coplanar slip systems form a laminate microstructure in which the separation between physical regions of single slip lacks dislocation walls which inhibit further slip. Thus, it is desirable to create a theory which combines coplanar slip regions into a single entity. Otherwise, hardening falsely occurs when a coplanar laminate microstructure activates further children systems. To understand this motivation for a coplanar model, it is required to understand the laminate model that is discussed in the following section (cf section 3); specifically refer to section 3.3.1 for details on coplanar dislocation structures. This section is placed here with minimal motivation to maintain a topical order to the paper.

In theories which include more traditional work hardening, it is apparent that coplanar hardening is much closer in magnitude to self-hardening than other forms of latent hardening (Bronkhorst 1991, Franciosi and Zaoui 1982, Havner 2005). Due to this hardening characteristic, coplanar slip hardening will be treated as a single self-hardening slip system.

Given the unique geometric nature of coplanar slip, multiple active coplanar slip systems can be treated as a single effective active slip system on a common plane. This is accomplished by defining an effective slip direction as a combination of the coplanar slip directions:

$$
{ }_{n} \hat{\boldsymbol{s}} \equiv \sum_{\alpha \in \text { coplanar }} \frac{{ }_{n} \dot{\gamma}_{\alpha}}{{ }_{n} \dot{\gamma}_{T}} s_{\alpha}
$$

where

$$
{ }_{n} \dot{\gamma}_{T} \equiv \sum_{\alpha \in \text { coplanar }} n \dot{\gamma}_{\alpha}
$$

Using this definition of $\hat{s}$, the values of the plastic deformation gradient, stress and plastic work energy (and thus, total energy) are equivalent to activating the slip systems simultaneously. Only the material tangent needs modification. These are shown subsequently.

As the coplanar slip model increases the degrees of freedom in the laminate, it creates new variables for the slip strains on each plane. These are solved by imposing the condition

$$
{ }_{n} \tau_{\alpha}={ }_{n} \tau_{\alpha}^{\mathrm{c}} \quad \text { for all } \alpha \in \text { coplanar. }
$$

Solving for the coplanar slip strains, ${ }_{n} \gamma_{\alpha}$, when this condition is met is simplified by considering that the minimum energy deformation in FCC crystals allows for a maximum of two slip systems to be active at any given time. Solving for the condition given by (2.36) requires a sequence of steps to implement as it is unknown a priori which slip systems activate or if single or multiple slip is active. The solution steps are as follows:

(i) Compute all $\tau_{\alpha}-\tau_{\alpha}^{\mathrm{c}}$ from (2.19) and (2.33).

(ii) Activate maximum $\tau_{\alpha}-\tau_{\alpha}^{\mathrm{c}}$ slip system from step i as single slip.

(iii) Recompute all $\tau_{\beta}-\tau_{\beta}^{\mathrm{c}}$ for all $\beta$ coplanar to $\alpha$ (to check if another coplanar slip system will activate after the first system)

if all $\tau_{\beta}-\tau_{\beta}^{\mathrm{c}} \leqslant 0$, exit (single slip system activated).

else, return max slip system to original slip strain and activate slip system $\alpha$ and highest coplanar $\tau_{\beta}-\tau_{\beta}^{\mathrm{c}}$, exit (two coplanar slip systems activated).

Computing coplanar slip using the single slip model requires a good initial guess for and an iteration over the effective slip direction. The initial guess $\hat{s}_{0}$, can be computed using the values of the initial shear stress on the slip systems, $\tau_{\alpha}-\tau_{\alpha}^{\mathrm{c}}$, and assuming $\boldsymbol{F}^{\mathrm{e}} \approx \boldsymbol{I}$ in (2.38). Note that the value of the derivative in (2.38) under this assumption is equal for all the slip directions since they are identical crystallographic directions.

$$
\hat{\boldsymbol{s}}_{0}=\frac{\left(\omega_{1}-\omega_{2} d\right)}{\left(\omega_{1}+\omega_{2}\right)(1-d)} \boldsymbol{s}_{1}+\frac{\left(\omega_{2}-\omega_{1} d\right)}{\left(\omega_{1}+\omega_{2}\right)(1-d)} \boldsymbol{s}_{2},
$$


where

$$
\begin{aligned}
& \omega_{\alpha}=\tau_{\alpha}-\tau_{\alpha}^{\mathrm{c}}, \\
& d=s_{1} \cdot s_{2} .
\end{aligned}
$$

Note that $d=1 / 2$ for FCC crystal systems.

Due to the approximation $\boldsymbol{F}^{\mathrm{e}} \approx \boldsymbol{I}$ this initial guess does not yield all $\tau_{\alpha}-\tau^{\mathrm{c}}=0$ for all active systems, $\alpha$, but can further be quickly driven to the exact solution by an iterative algorithm such as the Newton-Raphson. The required derivative for such an algorithm is

$$
\frac{\partial\left({ }_{n} \tau_{\alpha}-{ }_{n} \tau_{\alpha}^{\mathrm{c}}\right)}{\partial_{n} \gamma_{\beta}}=-\left({ }_{n} \boldsymbol{F}^{\mathrm{e}} \boldsymbol{s}_{\alpha} \otimes \boldsymbol{m}_{\alpha}\right) \cdot \mathcal{C}^{\mathrm{e}} \cdot\left({ }_{n} \boldsymbol{F}^{\mathrm{e}} \boldsymbol{s}_{\beta} \otimes \boldsymbol{m}_{\beta}\right)
$$

Note that '.' above sums over two indices as previously defined, hence the left-hand side is a scalar. The derivation of the above takes advantage of the fact that because $\boldsymbol{m} \cdot \boldsymbol{s}=0$ for all coplanar slip.

Using this definition of $\hat{s}$ in (2.34), the plastic work is equivalent to using the total slip strain:

$$
\Delta_{n} W^{\mathrm{p}}=\sum_{\alpha}\left({ }_{n} \tau_{\alpha}^{\mathrm{c}}\right)\left(\Delta_{n} \gamma_{\alpha}\right)
$$

where $\Delta_{n} \gamma_{\alpha} \equiv{ }_{n} \gamma_{\alpha}-{ }_{n-1} \gamma_{\alpha}$. Using the fact that all ${ }_{n} \tau_{\alpha}^{\mathrm{c}}$ are identical in a laminate with coplanar slip ((2.33) and (3.29)),

$$
\Delta_{n} W^{\mathrm{p}}=\left({ }_{n} \tau^{\mathrm{c}}\right)\left(\Delta_{n} \gamma_{T}\right)
$$

where $\Delta_{n} \gamma_{T} \equiv \sum_{\alpha \in \text { coplanar }} \Delta_{n} \gamma_{\alpha}$, which yields the desired result that the plastic work energy is equivalent.

For multiple activated coplanar systems, the plastic deformation gradient can also be treated with an equivalent slip direction. Since the slip in the following equations is coplanar, the subscript on $\boldsymbol{m}$ is dropped. For multiple simultaneous coplanar slip systems from (2.30)

$$
{ }_{n} \boldsymbol{F}_{l}^{\mathrm{p}}=\left(\boldsymbol{I}+\sum_{\alpha} \Delta_{n} \gamma_{\alpha} \boldsymbol{s}_{\alpha} \otimes \boldsymbol{m}\right)\left({ }_{n-1} \boldsymbol{F}_{l}^{\mathrm{p}}\right) .
$$

If instead a single slip system is activated on the plane $m$ with direction $\hat{s}$ and shear strain $\gamma_{T}$ :

$$
\begin{aligned}
& { }_{n} \boldsymbol{F}_{l}^{\mathrm{p}}=\left(\boldsymbol{I}+\Delta_{n} \gamma_{T} \hat{\boldsymbol{s}} \otimes \boldsymbol{m}\right)\left({ }_{n-1} \boldsymbol{F}_{l}^{\mathrm{p}}\right) \\
& =\left(\boldsymbol{I}+\left(\sum_{\alpha} \Delta_{n} \gamma_{\alpha} \boldsymbol{s}_{\alpha}\right) \otimes \boldsymbol{m}\right)\left({ }_{n-1} \boldsymbol{F}_{l}^{\mathrm{p}}\right) \\
& =\left(\boldsymbol{I}+\left(\sum_{\alpha} \Delta_{n} \gamma_{\alpha} \boldsymbol{s}_{\alpha} \otimes \boldsymbol{m}\right)\right)\left(_{n-1} \boldsymbol{F}_{l}^{\mathrm{p}}\right) .
\end{aligned}
$$

Note that if coplanar slip systems are activated sequentially it is also true that

$$
\begin{aligned}
{ }_{n} \boldsymbol{F}_{l}^{\mathrm{p}} & ={ }_{n} \boldsymbol{F}_{2}^{\mathrm{p}}{ }_{n} \boldsymbol{F}_{1}^{\mathrm{p}}\left({ }_{n-1} \boldsymbol{F}_{l}^{\mathrm{p}}\right) \\
& =\left(\boldsymbol{I}+{ }_{n} \gamma_{2} \boldsymbol{s}_{2} \otimes \boldsymbol{m}\right)\left(\boldsymbol{I}+{ }_{n} \gamma_{1} \boldsymbol{s}_{1} \otimes \boldsymbol{m}\right)\left({ }_{n-1} \boldsymbol{F}_{l}^{\mathrm{p}}\right) \\
& \left.=\left(\boldsymbol{I}+\left(\sum_{\alpha}{ }_{n} \gamma_{\alpha} \boldsymbol{s}_{\alpha} \otimes \boldsymbol{m}\right)\right){ }_{(n-1} \boldsymbol{F}_{l}^{\mathrm{p}}\right),
\end{aligned}
$$

where the last step in (2.43) recognizes that all cross terms in $\left(\sum_{\alpha} n \gamma_{\alpha} s_{\alpha}\right) \otimes \boldsymbol{m}$ and $\left(\boldsymbol{I}+{ }_{n} \gamma_{2} s_{2} \otimes \boldsymbol{m}\right)\left(\boldsymbol{I}+{ }_{n} \gamma_{1} \boldsymbol{s}_{1} \otimes \boldsymbol{m}\right)$ cancel due to $\boldsymbol{s} \cdot \boldsymbol{m}=0$ for coplanar systems.

The stress is unaffected since it is a function only of $\boldsymbol{F}$ and $\boldsymbol{F}^{\mathrm{p}}$. The material tangent for active coplanar slip does not conform to the single slip equation while using $\hat{s}$. The coplanar material tangent is

$$
\mathcal{C}_{i J k L}^{\text {coplanar }}=\mathcal{C}_{i M k N}^{\mathrm{e}}\left(F^{\mathrm{p}}\right)_{M J}^{-\mathrm{T}}\left(F^{\mathrm{p}}\right)_{N L}^{-\mathrm{T}}-\sum_{\alpha} \sum_{\beta} \frac{C_{i J \alpha}^{\text {mixed }} C_{k L \beta}^{\text {mixed }}}{\partial\left(\tau_{\beta}-\tau_{\beta}^{\mathrm{c}}\right) / \partial \gamma_{\alpha}},
$$


where the sum over $\alpha$ and $\beta$ is for the active slip systems, since by definition $\partial \gamma_{\alpha} / \partial\left(\tau_{\beta}-\tau_{\beta}^{\mathrm{c}}\right)=$ 0 if $\tau_{\beta}^{\mathrm{c}}>\tau_{\beta}$. In practice, it is more tractable to implement the single slip material tangent (2.27) using $\hat{s}$ for the slip direction. Although not exact, it still provides convergence within a reasonable number of iterations without excessive computation of $\boldsymbol{C}^{\text {mixed }}$ values. It should also be mentioned that if the active slip systems change, then there are corresponding discontinuities in the material tangent.

The preceding coplanar slip theory allows for the treatment of coplanar slip in a manner identical to that used for single slip. Note that the coplanar slip model will also produce any single slip condition as well.

\section{Laminate microstructures}

Following the previous work of Ortiz et al (2000), a formulation of laminate microstructures is used. Laminates are used due to the inability to resolve minimum energies for any general microstructure (Aubrey and Ortiz 2003), but the work of Ortiz and Repetto (1999) shows that many dislocation structures found in experiments can be reproduced using sequential laminate structures. The theory of sequential laminates used here is covered by Kohn (1991) and reviewed in other references (Ortiz and Repetto 1999, Aubrey and Ortiz 2003). The theory will be given here along with extensions to the nonlocal energy. Specifically dislocation mean free path length, evolution of laminate thickness, notes on the dislocation wall normals and coplanar slip are added to the theory.

Because the motivation of the coplanar slip model can only be understood after the dislocation wall energy is introduced, the significance of the zero-energy coplanar slip structures is discussed after the dislocation wall energy. In brief, since the coplanar slip laminate structures do not form dislocation walls to impede further dislocation motion, each spatial laminate instead of only activating a single slip system may activate a set of coplanar slip systems. The laminate structures are introduced using the coplanar slip model (cf section 2.3.4), which was shown to be used identically to the single slip model.

An advantage of using laminate structures is that complex structures can be formed by embedding simple laminate structures inside a previously formed laminate structure. A simple laminate is defined as a repetitive sequence of two material layers. As embedded simple laminate structures appear the same at any level, one only needs to describe and analyze the case of a single simple laminate, which can then be layered into more complicated structures. These are described as ranks by Kohn (1991). Each set of layers will be referred to as a 'laminate' (the use of node from previous works is changed to avoid future confusion with finite element nodes). Note that the term 'laminate' is not referring to a single laminate layer, but to a set of similar layers (e.g. the set of gray bands in figure 4 is one laminate). A laminate which is branched is called a 'branch', and the one which is not branched is called a 'leaf'. Each branch will have two 'children'. Every laminate except the rank 0 initial material will have one 'sibling' and one 'parent'. The notation followed for laminate designation is given in figure 3. The index $l$ will be used to refer to an unspecified laminate, with $l^{+}$and $l^{-}$being the children of $l$ and $l_{\mathrm{p}}$ being the parent of $l$. Note that each branch is identical, having one single slip system active in itself and dividing into a simple laminate structure with two slip systems. Hence, the complex laminate structure can be formed by an embedded composition of simple laminates. See figure 4 for an illustration of a grain with a rank two laminate subgrain structure. Note that rank 0 is the entire material.

Note that each laminate has only one active slip system (or set of coplanar slip systems treated as an effective single slip system), thus the single slip model is used for individual 


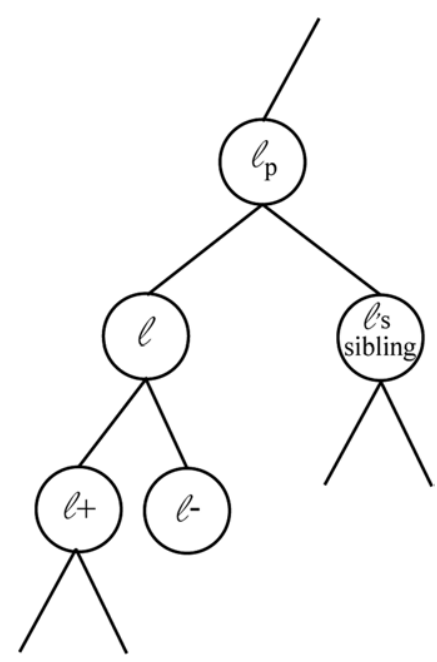

Figure 3. Labels used on laminate subgrain structures.
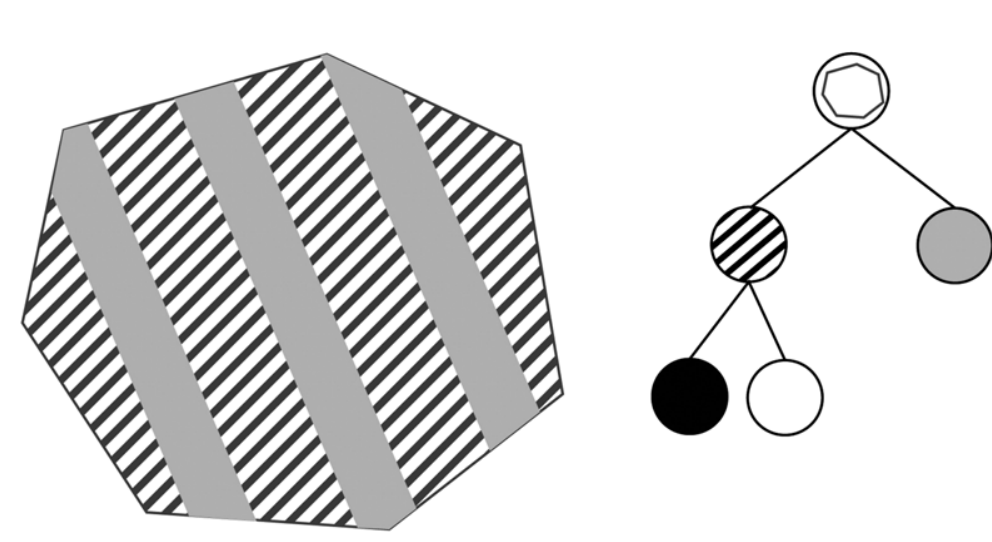

RANK

Figure 4. Illustration of a grain with a rank two subgrain laminate structure. Shading in the tree structure corresponds to the structure shown in the grain illustration.

laminate deformation. Also, since a laminate has only one active slip plane the index $\alpha$ used in the single slip section can be replaced by $l$ without modifying the equations.

A branched laminate is defined by the slip plane active on each side, $\alpha_{l^{ \pm}}$, combined laminate thickness, $L_{l}^{\mathrm{c}}$, the normal to the dislocation wall dividing the two laminates, $\boldsymbol{N}_{l}$, the volume fraction of each laminate, $\lambda_{l^{ \pm}}$, and the jump of the deformation across the wall, $\boldsymbol{a}_{l}$ (cf figure 5). One of the subtleties in the laminate theory is the evolution of the laminate parameters. These will be further discussed in the evolution section, but for ease of understanding the full work, those that evolve are given a time subscript $n$ in this section, namely ${ }_{n} L_{l}^{\mathrm{c}}$ and ${ }_{n} \boldsymbol{a}_{l}$. The parameters $\alpha_{l^{ \pm}}, N_{l}$ and $\lambda_{l^{ \pm}}$are not evolved.

Note that the slip system in a laminate cannot change at later time steps. Also, the amount of slip deformation of a branched laminate is not evolved. Thus, once a laminate forms, it always remains in the microstructure although it can further branch itself. This provides the theory with path dependence. To extend the loading path dependence for all conditions it would be required to allow for dissolution of laminate walls. Although dissolution is not considered 


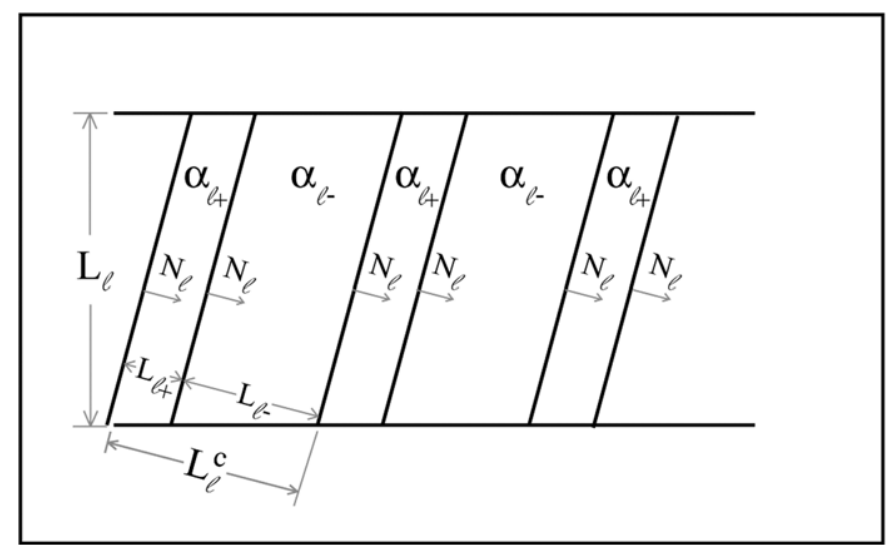

Figure 5. Simple laminate structure variables. Illustration is not to scale.

in this work, it should be relatively straightforward to add in dissolution of the finest layer of laminates formed if the slip systems involved reverse; dissolution of higher rank laminates would require a model relating reloading and dissolution of structure.

The framework for the laminate structures needs to be presented before nonlocal energy terms can be understood, but the nonlocal terms will affect the laminate framework. Rather than showing the general equations for the laminates and then modifying them later to contain the nonlocal terms, the nonlocal terms are included without a full explanation and derived in the next section. Equilibration, discussed last, now includes the laminate thickness providing an accurate form of evolving slip hardening.

\subsection{Laminate variable properties}

As the deformation must be rank one connected

$$
{ }_{n} \boldsymbol{F}_{l^{+}}-{ }_{n} \boldsymbol{F}_{l^{-}}={ }_{n} \boldsymbol{a}_{l} \otimes \boldsymbol{N}_{l},
$$

where $\left\{{ }_{n} a_{l}, N_{l}\right\} \in \mathfrak{R}^{3}$ and as $\boldsymbol{N}_{l}$ is a unit vector indicating direction, $\|N\|=1$. The symbol ${ }_{n} \boldsymbol{a}_{l}$ is the polarization vector which varies with the values of the deformation gradient.

Obviously, the volume fractions, $\lambda_{l} \in \Re$. Note that $\lambda_{l}$ must obey

$$
\lambda_{l^{+}}+\lambda_{l^{-}}=1, \quad \lambda_{l^{ \pm}} \in[0,1] .
$$

Given the volume fraction, the deformation of the parent laminate follows as

$$
{ }_{n} \boldsymbol{F}_{l}=\left(\lambda_{l^{-}}\right)\left({ }_{n} \boldsymbol{F}_{l^{-}}\right)+\left(\lambda_{l^{+}}\right)\left({ }_{n} \boldsymbol{F}_{l^{+}}\right) .
$$

From (3.1) and (3.3), the deformation gradient of the children can be written as

$$
{ }_{n} \boldsymbol{F}_{l^{ \pm}}=\left({ }_{n} \boldsymbol{F}_{l}\right) \pm \lambda_{l^{\mp}}\left({ }_{n} \boldsymbol{a}_{l} \otimes \boldsymbol{N}_{l}\right) .
$$

Aubrey and Ortiz (2003), Ortiz et al (2000), Ortiz and Repetto (1999) and Ortiz and Stainier (1999) derive the relationship

$$
{ }_{n} \boldsymbol{P}_{l}=\left(\lambda_{l^{-}}\right)\left({ }_{n} \boldsymbol{P}_{l^{-}}\right)+\left(\lambda_{l^{+}}\right)\left({ }_{n} \boldsymbol{P}_{l^{+}}\right),
$$

and the material tangent for a branched laminate

$$
{ }_{n} \mathcal{C}_{l}^{\text {laminate }}=\left(\lambda_{l^{+}}\right)\left({ }_{n} \mathcal{C}_{l^{+}}^{\text {s.s. }}\right)+\left(\lambda_{l^{-}}\right)\left({ }_{n} \mathcal{C}_{l^{-}}^{\text {s.s. }}\right),
$$

where equation (2.27) is used to calculate ${ }_{n} \mathcal{C}_{l^{ \pm}}^{\text {s.s. }}$. 
The characteristic width of a laminate's layers is the combined width of the two children's layers:

$$
{ }_{n} L_{l}^{\mathrm{c}} \equiv{ }_{n} L_{l+}+{ }_{n} L_{l-} .
$$

Due to the repetitive planar geometry of a laminate structure, the width of a child laminate's layers is related to its parent layer's width by the volume fraction

$$
{ }_{n} L_{l^{ \pm}}=\lambda_{l^{ \pm}}{ }_{n} L_{l}^{\mathrm{c}} \text {. }
$$

A laminate's characteristic width, ${ }_{n} L_{l}^{\mathrm{c}}$, is further restricted to be less than the width of the parent structure:

$$
{ }_{n} L_{l}^{\mathrm{c}} \leqslant{ }_{n} L_{l_{\mathrm{p}}} .
$$

Note that this restriction has been added as it was found that energy minimization alone allowed for the nonphysical case of children larger than the parent.

When a laminate branches and becomes a parent the plastic deformation that has occurred in the parent during previous time steps is retained as this deformation is permanent and has already occurred. The children accommodate any remaining deformation. The current plastic deformation in the parent (e.g. ${ }_{n-1} \boldsymbol{F}_{l}^{\mathrm{p}}$ ) will not continue to evolve with further deformation after the children laminates are formed. However, the parent's total deformation, ${ }_{n} \boldsymbol{F}_{l}$, will evolve as the children continue deforming ( $\mathrm{cf}(3.3)$ ). In order to account for the previous plastic deformation when a laminate forms, its previous time step's plastic deformation is set equal to its parent's previous plastic deformation (given for the + child, but with the - child analogous):

$$
{ }_{n-1} \boldsymbol{F}_{l^{+}}^{\mathrm{p}}={ }_{n-1} \boldsymbol{F}_{l}^{\mathrm{p}},
$$

so that by equation (2.30)

$$
{ }_{n} \boldsymbol{F}_{l^{+}}^{\mathrm{p}}=\left(\boldsymbol{I}+\Delta \gamma_{l^{+}} \hat{\boldsymbol{s}}_{l^{+}} \otimes \boldsymbol{m}_{l^{+}}\right)_{n-1} \boldsymbol{F}_{l}^{p},
$$

and the correct elastic deformation is given by equation (2.6), ${ }_{n} \boldsymbol{F}_{l^{+}}^{\mathrm{e}}={ }_{n} \boldsymbol{F}_{0}\left({ }_{n} \boldsymbol{F}_{l^{+}}^{\mathrm{p}}\right)^{-1}$, where ${ }_{n} \boldsymbol{F}_{0}$ is the global deformation, e.g. $F$ at time $t_{n}$ in the laminate at rank 0 . The previous slip strain of the children is set to zero, ${ }_{n-1} \gamma_{l^{+}}=0$, so that the incremental plastic energy is correctly computed (2.40).

\subsection{Branching energy computation}

The minimum energy laminate structure should be found in order to make comparisons with the single slip and elastic energies. Thus, one finds the solution to

$$
\begin{aligned}
{ }_{n} W_{l}\left[{ }_{n} \boldsymbol{F}_{l}\right] \equiv \min _{\alpha_{l^{ \pm},{ }_{n}} \boldsymbol{a}_{l}, \boldsymbol{N}_{l}, \lambda_{l^{ \pm},{ }_{n}} L_{l}^{\mathrm{c}}} & \left(\lambda_{l^{-}}{ }_{n} W_{l^{-}}\left[\alpha_{l^{-}},{ }_{n} \boldsymbol{a}_{l}, \boldsymbol{N}_{l},{ }_{n} L_{l}^{\mathrm{c}}\right]+\lambda_{l^{+}}{ }_{n} W_{l^{+}}\left[\alpha_{l^{+}},{ }_{n} \boldsymbol{a}_{l}, \boldsymbol{N}_{l},{ }_{n} L_{l}^{\mathrm{c}}\right]\right. \\
+ & \left.\left({ }_{(n-1)} \tau_{l}\right)\left({ }_{(n-1)} \gamma_{l}\right)+2 \Upsilon \frac{{ }_{n} L_{l}^{\mathrm{c}}}{{ }_{n} L_{l}}{ }_{n} W_{l}^{\mathrm{BL}}\left[\alpha_{l^{ \pm}},{ }_{n} \boldsymbol{a}_{l}, \boldsymbol{N}_{l}, \lambda_{l^{ \pm}},{ }_{n} L_{l}^{\mathrm{c}}\right]\right) .
\end{aligned}
$$

This is the minimum energy density of laminate $l$ branching and becoming any two leaves $l^{+}$ and $l^{-}$. If this energy density is less than the energy density of activating a slip plane (coplanar slip), the laminate forms. Purely elastic leaves are excluded from consideration. The first term is the energy density from the laminate $l^{-}$, both the elastic $W^{\mathrm{e}}$ and plastic $W^{\mathrm{p}}$. The second term is likewise the energy density from laminate $l^{+}$. The third term is the plastic energy that the possibly branching laminate $l$ has already stored by plastic coplanar slip; it is included in the 
branched energy in order to make accurate comparisons with the energy density of continuing to slip on a single slip plane. Note that the energy accumulated in this term has already been completed in the previous deformation step, and due to the assumed irreversibility of slip it does not evolve further; thus, it can be removed from this minimization as a constant. The fourth term is the boundary layer energy caused by the misfit between the laminates and the boundaries of the parent system. $\Upsilon$ is the depth of the boundary layer. Readers may refer to section 3.3.2 for details on the boundary layer energy density. The [.] shows the functional dependence on the variables varied in the minimization.

Note that the previous work of Aubrey and Ortiz (2003) treated the nonlocal energy terms of the boundary layer and the dislocation walls as perturbations of the energy, and hence were not directly included in the minimization (3.12). When the nonlocal energy is introduced later, it will be estimated to show that it should not be treated as a perturbation, but minimized directly.

The slip systems, $\alpha_{l^{ \pm}}$, wall normal, $\boldsymbol{N}_{l}$, and volume fraction, $\lambda_{l}$, of each possible branch have well-defined domains which are searched. The values for the jump vector, $\boldsymbol{a}_{l}$, and the length scale of the laminate, $L_{l}$, each have a direct minimization equation if the other parameters are specified. There is an interaction between $\boldsymbol{a}_{l}$ and $L_{l}$, which is iterated over to obtain a minimum. To find the minimum energy branched structure the values of $\alpha_{l^{ \pm}}, N_{l}$ and $\lambda_{l}$ are iterated over for all possible values solving for $\boldsymbol{a}_{l}$ and $L_{l}$ by minimization within each possible set. The lowest energy of all possible parameter values is selected for comparison with single (coplanar) slip. Full details for each parameter follow.

3.2.1. Slip plane selection, $\alpha$. The possible slip planes will be iterated over to find a minimum deformation energy with respect to $\alpha_{l^{ \pm}}$. This reduces the required solution of (3.12) to finding

$$
\begin{aligned}
{ }_{n} W_{l}\left[{ }_{n} \boldsymbol{F}_{l}\right]_{\alpha_{l^{ \pm}}^{*}}= & \left({ }_{(n-1)} \tau_{l}\right)\left({ }_{(n-1)} \gamma_{l}\right)+{ }_{{ }_{n} \boldsymbol{a}_{l}, \boldsymbol{N}_{l}, \lambda_{l^{ \pm},{ }_{n} L_{l}^{\mathrm{c}}}} \\
& \left(\lambda_{l^{-}}{ }_{n} W_{l^{-}}\left[\alpha_{l^{-}}^{*},{ }_{n} \boldsymbol{a}_{l}, \boldsymbol{N}_{l},{ }_{n} L_{l}^{\mathrm{c}}\right]+\lambda_{l^{+}}{ }_{n} W_{l^{+}}\left[\alpha_{l^{+}}^{*},{ }_{n} \boldsymbol{a}_{l}, \boldsymbol{N}_{l},{ }_{n} L_{l}^{\mathrm{c}}\right]\right. \\
+ & \left.2 \Upsilon \frac{{ }_{n} L_{l}^{\mathrm{c}}}{{ }_{n}{ }_{l}}{ }_{n} W_{l}^{\mathrm{BL}}\left[\alpha_{l^{ \pm}}^{*},{ }_{n} \boldsymbol{a}_{l}, \boldsymbol{N}_{l}, \lambda_{l^{ \pm},{ }_{n}} L_{l}^{\mathrm{c}}\right]\right),
\end{aligned}
$$

for a given set of two slip planes $\alpha_{l^{+}}^{*}$ and $\alpha_{l^{-}}^{*}$ using the given restrictions.

3.2.2. Dislocation wall normals, $\boldsymbol{N}$. The complexity of calculating the wall normal when only single slip (not coplanar) is active can be reduced by referring to work done by Ortiz and Repetto (1999), although care must be taken in how these are implemented. Ortiz and Repetto tabulated wall normals using rank one compatibility (3.1) of deformation assuming only plastic single slip on each side of a rank one laminate with no elastic deformation for FCC crystal systems. Under the plausible assumption that near the dislocation wall the majority of deformation is plastic, as little distance exists for elastic deformation, these derived plastic deformation wall normals can be assumed a good approximation. However, two details must be considered when implementing these directly from the reference. First, they were derived in the Eulerian reference frame and second, the length evolution of the wall normal for degenerate cases, i.e. those cases for which the slip system normals or slip system directions are identical. Note that in the derivation of the wall normals there is also a restriction placed on the ratio $\dot{\gamma}^{+} / \dot{\gamma}^{-}$, which is not directly applied in this work. This again is related to the assumption that all the deformation contained in the compatible wall is plastic, while elsewhere, elastic deformation eases any restriction. 
The conversion of the Lagrangian reference frame normal $N$ to the Eulerian reference frame normal $\boldsymbol{n}$ is given in Ortiz and Repetto (1999):

$$
\boldsymbol{n}=\frac{\left(\boldsymbol{F}_{l^{+}}\right)^{-\mathrm{T}} \boldsymbol{N}_{l}}{\left\|\left(\boldsymbol{F}_{l^{+}}\right)^{-\mathrm{T}} \boldsymbol{N}_{l}\right\|},
$$

This transformation is discussed in detail in Hansen (2009).

From Ortiz and Repetto (1999), the branched laminate composed of the two single slip systems $\beta_{l^{+}}$and $\beta_{l^{-}}$has only two possible wall normal orientations if the slip systems are not degenerate, i.e. the slip directions are not identical or the slip planes are not identical. These two possibilities for wall normal direction can then be iterated over and only the lower energy taken.

For the degenerate case in which the slip directions are equal it can be shown that

$$
\boldsymbol{n}_{l}=\frac{\dot{\gamma}_{l^{+}} \boldsymbol{m}_{l^{+}}-\dot{\gamma}_{l^{-}} \boldsymbol{m}_{l^{-}}}{\left\|\dot{\gamma}_{l^{+}} \boldsymbol{m}_{l^{+}}-\dot{\gamma}_{l^{-}} \boldsymbol{m}_{l^{-}}\right\|} .
$$

Thus, the normal belongs to a 2D unit circle which contains $\boldsymbol{m}_{l^{+}}$and $\boldsymbol{m}_{l^{-}}$. This unit circle is iterated over by taking a set angle interval. The normal with the lowest energy is selected. Note since the opposite of the normal gives an identical wall orientation, only half of the circle needs to be checked. (If $N_{l} \rightarrow-N_{l}$, then $\boldsymbol{a}_{l} \rightarrow-\boldsymbol{a}_{l}$, and the calculations are not affected.) Also, the directions $\boldsymbol{m}^{+}$and $\boldsymbol{m}^{-}$may be excluded as those directions correspond to only activating one of the slip planes.

For the degenerate cases in which the slip plane normals are identical or coplanar slip,

$$
n_{l}=m_{l^{+}}=m_{l^{-}} .
$$

Due to the parallel slip planes the dislocations of these two systems will not interact and will not form a dislocation wall. This and other considerations for the wall normal in this section were noted by Ortiz and Repetto (1999), but were not considered in the initial theory of Aubrey and Ortiz (2003). Coplanar slip systems have already been discussed in section 2.3.4 and will be discussed further in section 3.3.1. It should be noted here that the dislocation wall is parallel to the slip planes for coplanar slip. It will be shown that due to this condition these systems are not resolved in the energy calculations.

Unfortunately, these conditions hold only for single slip. For considering the formation of laminates in which more than one slip system is activated on one side of the wall (e.g. coplanar slip systems), there is presently no alternative but to search the unit sphere of all wall normals for the lowest energy wall normal.

As described, the set of all possible $\boldsymbol{N}_{l}$ can now be iterated over, thus reducing the minimization of (3.13) to finding the value for a given $\boldsymbol{N}_{l}^{*}$. While considering single slip activation (not coplanar), the set of possible wall normals considered is reduced using compatibility.

3.2.3. Computation of volume fraction, $\lambda$. As there are strict bounds limiting $\lambda$ to the domain $\lambda \in[0,1]$, a discrete minimization of $\lambda$ can be conducted. Note that the endpoints of the domain can be avoided as they pertain to a single volume of uniform material. Using an accuracy of $0.1, \lambda_{l}$ is given the possible set of values

$$
\lambda_{l} \in\{0.1,0.2,0.3,0.4,0.5,0.6,0.7,0.8,0.9\} .
$$

All that is lost in this approach of solving for $\lambda$ is accuracy in the volume fraction of the laminate.

It is possible that a more computationally efficient solution would simultaneously solve for $\lambda, a$ and $L^{\mathrm{c}}$, but the discrete optimization is much more timely to implement. 
The minimization (3.13) is now reduced to

$$
\begin{aligned}
{ }_{n} W_{l}\left[{ }_{n} \boldsymbol{F}_{l}\right]_{\alpha_{l^{ \pm}}, N_{l}^{*}, \lambda_{l^{ \pm}}^{*}}=\left({ }_{(n-1)} \tau_{l}\right)\left({ }_{(n-1)} \gamma_{l}\right)+\min _{n} \boldsymbol{a}_{l, n} L_{l}^{\mathrm{c}} & \left(\lambda_{l^{-} n}^{*} W_{l^{-}}\left[\alpha_{l^{-}}^{*},{ }_{n} \boldsymbol{a}_{l}, \boldsymbol{N}_{l}^{*},{ }_{n} L_{l^{\mathrm{c}}}\right]+\lambda_{l^{+}{ }_{n}}^{*} W_{l^{+}}\left[\alpha_{l^{+}}^{*},{ }_{n} \boldsymbol{a}_{l}, \boldsymbol{N}_{l}^{*},{ }_{n} L_{l}^{\mathrm{c}}\right]\right. \\
+ & \left.2 \Upsilon \frac{{ }_{n} L_{l}^{\mathrm{c}}}{{ }_{n}{ }_{l}}{ }_{n} W_{l}^{\mathrm{BL}}\left[\alpha_{l^{ \pm}}^{*},{ }_{n} \boldsymbol{a}_{l}, \boldsymbol{N}_{l}^{*}, \lambda_{l^{ \pm}}^{*},{ }_{n} L_{l}^{\mathrm{c}}\right]\right) .
\end{aligned}
$$

3.2.4. Computation of polarization vector, $\boldsymbol{a}$. Minimization of (3.17) excluding the boundary layer energy with respect to $a_{l}$ while holding ${ }_{n} L_{l}^{\mathrm{c}}$ constant $\left({ }_{n} L_{l}^{\mathrm{c}}={ }_{n} L_{l}^{\mathrm{c} *}\right)$ gives the condition that tractions must be compatible across dislocation walls:

$$
\left({ }_{n} \boldsymbol{P}_{l^{+}}-{ }_{n} \boldsymbol{P}_{l^{-}}\right) \boldsymbol{N}_{l}=\mathbf{0} .
$$

This is solved directly using a Newton-Raphson iteration for the value of $\boldsymbol{a}_{l}$. It will be shown that generally $W_{l}^{\mathrm{BL}}$ is small compared with the total plastic energy.

This minimization ideally should be done concurrently with the minimization of ${ }_{n} L_{l}^{\mathrm{c}}$ as both affect the plastic energy, but currently a loop over the solution of ${ }_{n} a_{l}$ and ${ }_{n} L_{l}^{\mathrm{c}}$ is performed until convergence of ${ }_{n} L_{l}^{\mathrm{c}}$ is reached. Convergence could be more efficient by the introduction of a minimization solution over ${ }_{n} \boldsymbol{a}_{l}$ and ${ }_{n} L_{l}^{\mathrm{c}}$ simultaneously. This analysis is difficult as the iteration in solving for ${ }_{n} \gamma_{l^{ \pm}}$prevents an analytical equation.

3.2.5. Computation of laminate thickness, $L^{\mathrm{c}}$. With the given values for $\alpha_{l}^{ \pm}, \lambda_{l}$ and $N_{l}$ and the last iterative solution ${ }_{n} \boldsymbol{a}_{l}={ }_{n} \boldsymbol{a}_{l}^{*}$, the solution for ${ }_{n} L_{l}^{\mathrm{c}}$ is a simple analytical minimization. The value ${ }_{n} L_{l}^{\mathrm{c}}$ appears only in the nonlocal terms as an addition to the critical resolved shear stress in the plastic energy and in the boundary layer energy. (The nonlocal energy is explained subsequently, but the minimization solution is placed here to be included with the other laminate parameter calculations.) The thickness of the laminates is derived through a competition between the two nonlocal energies.

Breaking up ${ }_{n} W_{l^{-}}$using in turn (2.31), (2.32) and (2.33) yields

$$
\begin{aligned}
{ }_{n} W_{l^{-}} & ={ }_{n} W_{l^{-}}^{\mathrm{e}}+{ }_{n} W_{l^{-}}^{\mathrm{p}} \\
& ={ }_{n} W_{l^{-}}^{\mathrm{e}}+\left({ }_{n} \tau_{l^{-}}^{\mathrm{c}}\right)\left({ }_{n} \gamma_{l^{-}}\right) \\
& ={ }_{n} W_{l^{-}}^{\mathrm{e}}+\left({ }_{\tau_{0}}^{\mathrm{c}}\right)\left({ }_{n} \gamma_{l^{-}}\right)+\frac{T}{b_{n} h_{l^{-}}}{ }_{n} \gamma_{l^{-}} .
\end{aligned}
$$

Using (3.31b) (the definition of dislocation wall energy density, $W^{\mathrm{DW}}$ ) and (3.19)

$\lambda_{l^{-}}{ }_{n} W_{l^{-}}+\lambda_{l^{+}}{ }_{n} W_{l^{+}}=\lambda_{l^{-}}\left({ }_{n} W_{l^{-}}^{\mathrm{e}}+\tau_{0}^{\mathrm{c}}\left({ }_{n} \gamma_{l^{-}}\right)\right)+\lambda_{l^{+}}\left({ }_{n} W_{l^{+}}^{\mathrm{e}}+\tau_{0}^{\mathrm{c}}\left({ }_{n} \gamma_{l^{+}}\right)\right)+{ }_{n} W_{l} \mathrm{DW}$

Using the values of ${ }_{n} \gamma_{l^{ \pm}}$and ${ }_{n} W_{l^{ \pm}}^{\mathrm{e}}$ calculated during the minimization of ${ }_{n} a_{l}$ with ${ }_{n} L_{l}^{\mathrm{c}}={ }_{n} L_{l}^{\mathrm{c} *}$, the dependences can be written as

$$
\begin{aligned}
& \lambda_{l^{-}{ }_{n}} W_{l^{-}}\left[\alpha_{l^{-}}^{*},{ }_{n} \boldsymbol{a}_{l^{*}}, \boldsymbol{N}_{l}^{*},{ }_{n} L_{l}^{\mathrm{c} *},{ }_{n} L_{l}^{\mathrm{c}}\right]+\lambda_{l^{+}}{ }_{n} W_{l^{+}}\left[\alpha_{l^{+}}^{*},{ }_{n} \boldsymbol{a}_{l}{ }^{*}, \boldsymbol{N}_{l}^{*},{ }_{n} L_{l}^{\mathrm{c} *},{ }_{n} L_{l}^{\mathrm{c}}\right] \\
& =\lambda_{l^{-}}\left({ }_{n} W_{l^{-}}^{\mathrm{e}}\left[\alpha_{l^{-}}^{*},{ }_{n} \boldsymbol{a}_{l^{*}}, \boldsymbol{N}_{l}^{*}\right]+\tau_{0}^{\mathrm{c}}\left({ }_{n} \gamma_{l^{-}}\left[\alpha_{l^{-}}^{*},{ }_{n} \boldsymbol{a}_{l^{*}}, \boldsymbol{N}_{l}^{*},{ }_{n} L_{l}^{\mathrm{c} *}\right]\right)\right) \\
& +\lambda_{l^{+}}\left({ }_{n} W_{l^{+}}^{\mathrm{e}}\left[\alpha_{l^{+}}^{*},{ }_{n} \boldsymbol{a}_{l^{*}}{ }^{*}, \boldsymbol{N}_{l}^{*}\right]+\tau_{0}^{\mathrm{c}}\left({ }_{n} \gamma_{l^{+}}\left[\alpha_{l^{+}}^{*},{ }_{n} \boldsymbol{a}_{l}{ }^{*}, \boldsymbol{N}_{l}^{*},{ }_{n} L_{l}^{\mathrm{c} *}\right]\right)\right) \\
& +{ }_{n} W_{l}^{\mathrm{DW}}\left[\alpha_{l^{ \pm}}^{*},{ }_{n} \boldsymbol{a}_{l^{*}}, \boldsymbol{N}_{l}^{*},{ }_{n} L_{l}^{\mathrm{c}}\right] \\
& =W^{*}\left[\alpha_{l^{-}}^{*},{ }_{n} \boldsymbol{a}_{l^{*}}, \boldsymbol{N}_{l}^{*},{ }_{n} L_{l}^{\mathrm{c} *}\right]+{ }_{n} W_{l}^{\mathrm{DW}}\left[\alpha_{l^{ \pm}}^{*},{ }_{n} \boldsymbol{a}_{l}{ }^{*}, \boldsymbol{N}_{l}^{*},{ }_{n} L_{l}^{\mathrm{c}}\right],
\end{aligned}
$$

where $W^{*}=\lambda_{l^{-}}\left({ }_{n} W_{l^{-}}^{\mathrm{e}}+\left(\tau_{0}^{\mathrm{c}}\right)\left({ }_{n} \gamma_{l^{-}}\right)\right)+\lambda_{l^{+}}\left({ }_{n} W_{l^{+}}^{\mathrm{e}}+\left(\tau_{0}^{\mathrm{c}}\right)\left({ }_{n} \gamma_{l^{+}}\right)\right)$is introduced for brevity. 
With this notation the minimization is

$$
\begin{aligned}
{ }_{n} W_{l}\left[{ }_{n} \boldsymbol{F}_{l}\right]_{\alpha_{l^{ \pm}}, N_{l}^{*}, \lambda_{l^{ \pm}}^{*}, a_{l}^{*}}=\left({ }_{(n-1)} \tau_{l}\right)\left({ }_{(n-1)} \gamma_{l}\right) \\
\quad+\min _{n}\left(W^{*}\left[\alpha_{l}^{*},{ }_{n} \boldsymbol{a}_{l^{*}}{ }^{*}, \boldsymbol{N}_{l}^{*},{ }_{n} L_{l}^{c *}\right]+{ }_{n} W_{l}^{\mathrm{DW}}\left[\alpha_{l^{ \pm}}^{*},{ }_{n} \boldsymbol{a}_{l^{*}}{ }^{*}, \boldsymbol{N}_{l}^{*},{ }_{n} L_{l}^{\mathrm{c}}\right]\right. \\
\left.+2 \Upsilon \frac{{ }_{n} L_{l}^{\mathrm{c}}}{L_{l}}{ }_{n} W_{l}^{\mathrm{BL}}\left[\alpha_{l^{ \pm}}^{*},{ }_{n} \boldsymbol{a}_{l}, \boldsymbol{N}_{l}^{*}, \lambda_{l^{ \pm}}^{*},{ }_{n} L_{l}^{\mathrm{c}}\right]\right) \\
=\left({ }_{(n-1)} \tau_{l}\right)\left({ }_{(n-1)} \gamma_{l}\right)+W^{*}+\min _{{ }_{n} L_{l}^{\mathrm{c}}}\left({ }_{n} W_{l}^{\mathrm{DW}}+2 \Upsilon \frac{{ }_{n} L_{l}^{\mathrm{c}}}{{ }_{n-1} L_{l}}{ }_{n} W_{l}^{\mathrm{BL}}\right) .
\end{aligned}
$$

Thus, the minimization with respect to ${ }_{n} L_{l}^{\mathrm{c}}$ is only over the nonlocal energy density, ${ }_{n} W_{l}^{\mathrm{NL}}$ (3.35). Substituting for the dislocation wall energy (3.31b),

$$
{ }_{n} W_{l}^{\mathrm{NL}}=\frac{T}{b_{n} L_{l}^{\mathrm{c}}}\left(\frac{n \gamma_{l^{-}}}{\zeta_{l^{-}}} \sqrt{1-\left(\boldsymbol{m}_{l^{-}} \cdot \boldsymbol{N}_{l}\right)^{2}}+\frac{{ }_{n} \gamma_{l^{+}}}{\zeta_{l^{+}}} \sqrt{1-\left(\boldsymbol{m}_{l^{+}} \cdot \boldsymbol{N}_{l}\right)^{2}}\right)+2 \Upsilon \frac{{ }_{n} L_{l}^{\mathrm{c}}}{{ }_{n} L_{l}}{ }_{n} W_{l}^{\mathrm{BL}},
$$

where $\zeta_{l}$ is the ratio of the dislocation mean free path length to the width of the laminate; it will be introduced in section 3.3.1.

The minimization with respect to ${ }_{n} L_{l}^{\mathrm{c}}$ then follows as

${ }_{n} L_{l}^{\mathrm{c}}=\sqrt{\frac{T_{n} L_{l}}{2 \Upsilon b_{n} W_{l}^{\mathrm{BL}}}\left(\frac{n \gamma_{l^{-}}}{\zeta_{l^{-}}} \sqrt{1-\left(\boldsymbol{m}_{l^{-}} \cdot \boldsymbol{N}_{l}\right)^{2}}+\frac{n \gamma_{l^{+}}}{\zeta_{l^{+}}} \sqrt{1-\left(\boldsymbol{m}_{l^{+}} \cdot \boldsymbol{N}_{l}\right)^{2}}\right) .}$

Adding in the constraint that the children fit inside the parent

$$
{ }_{n} L_{l}^{\mathrm{c}}= \begin{cases}\text { equation }(3.24) & \text { if equation }(3.24)<{ }_{n-1} L_{l}, \\ { }_{n-1} L_{l} & \text { otherwise. }\end{cases}
$$

This work minimizes the nonlocal energy directly, as it will be shown in section 3.3.1 that the dislocation wall energy will eventually be comparable in magnitude to the plastic deformation energy giving a strong interaction between $L_{l}^{\mathrm{c}}$ and $a_{l}$.

\subsection{Nonlocal energy}

Following the ideas of Aubrey and Ortiz (2003), two terms of nonlocal energy will be included due to the formation of laminate structures: the dislocation wall energy and the boundary layer energy.

3.3.1. Dislocation wall energy. When a laminate branches into two laminates with different slip planes, a dislocation wall forms between them. The new dislocation walls impede dislocation motion and, thus, shorten the mean free path of the mobile dislocations. As derived in Aubrey and Ortiz (2003) using Orowan's relation, shortening the mean free path causes plastic energy for a given deformation to increase. This increase in the plastic deformation energy corresponds to an increase in critical resolved shear stress. Hence the formation of dislocation walls increases the energy necessary for further plastic deformation for the individual laminate's slip system, but due to the gained freedom in active slip systems to release stored elastic energy the laminate formation may lower the global deformation energy.

Here the ideas of Aubrey and Ortiz (2003) are expanded to not just using the width of the laminate as the mean free path for dislocations, but to include the angle between the path of the dislocation and the dislocation wall. This allows a first estimate of morphology in the structure to be included. The inclusion of the dislocation path geometry means finer laminates will form if the slip plane (and thus the dislocation paths) is nearly parallel to the dislocation wall. This 


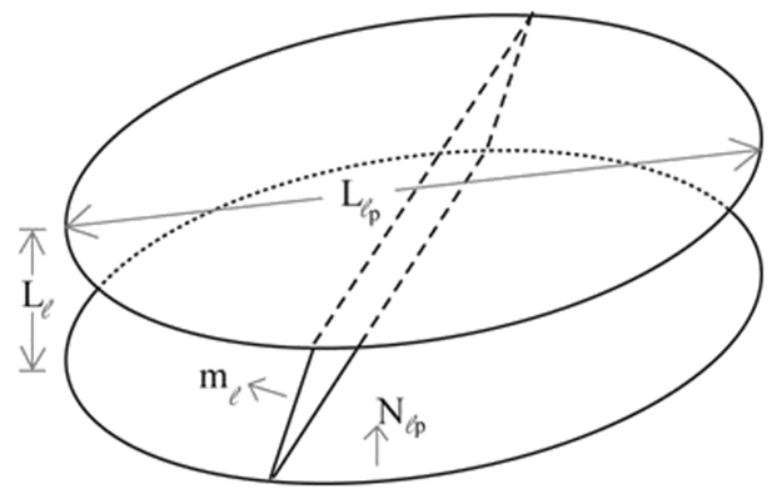

Figure 6. Geometry of slip plane between two dislocation walls.

change significantly lowered the boundary layer energy and often changed the lowest energy (active) system in simulations. This is particularly important for the special case of coplanar slip where the dislocation path normals in sibling laminate structures are identical and thus, no dislocation walls form to impede further dislocation slip (Ortiz and Repetto 1999).

For a given slip system $\beta$, dislocations are assumed to move until they encounter the boundary of the laminate. Note that all dislocation paths reside on the slip plane regardless of the type of dislocation; thus one needs to consider the general case of computing the mean free path of dislocations on the slip plane of laminate $l$ which is between two parallel dislocation walls (see figure 6). The width between the parallel dislocation walls, $L_{l}$, is defined by the computation of $L_{l_{\mathrm{p}}}^{\mathrm{c}}$ and $\lambda_{l}$ from (3.8). The length of the dislocation walls is approximated as the width of the parent laminate: $L_{l_{\mathrm{p}}}$. Note that this approximation is a minimum; the true length may be much longer depending on the angle the parent laminate makes with its parent and grandparent and so on. This is not of concern though as a separation of scales is assumed, e.g. $L_{l_{\mathrm{p}}} \gg L_{l_{\mathrm{p}}}^{\mathrm{c}}$. As this assumption will be used for simplification, it is not of any consequence if $L_{l_{\mathrm{p}}}$ is underestimated. The normal to the dislocation walls is $\boldsymbol{N}_{l_{\mathrm{p}}}$ and the normal to the slip plane is $\boldsymbol{m}_{l}$.

The slip plane is approximated as a rectangle defined by two lengths, call the lengths $A$ and $B$ defined by

$$
\begin{aligned}
A & =\frac{L_{l}}{\sqrt{1-\left(\boldsymbol{m}_{l} \cdot N_{l_{\mathrm{p}}}\right)^{2}}}, \\
B & =L_{l_{\mathrm{p}}} .
\end{aligned}
$$

Table 2 lists the ratios $L / A$ for the slip system A6 given the sibling slip system for single slip using all possible wall normals as calculated by Ortiz and Repetto (1999). Due to symmetries of the slip systems it does not matter which system was chosen; A6 is a completely generic choice. Note that microstructures involving only the same slip system (i.e. A6 and A6 in this example) are indistinguishable in this theory. The value of $L / A$ ranges from a maximum of 1 (slip plane perpendicular to laminate wall normal) to a minimum of $\frac{1}{3}$, excluding the same slip plane laminates. The same slip plane laminates (i.e. A slip systems in this example) are parallel to the dislocation wall normal and thus do not interact with it. These are discussed in section 3.3.1 subsequently. 
Table 2. $L / A$ ratios for generic slip system A6.

\begin{tabular}{|c|c|c|}
\hline System & $n$ (not normalized) & $\frac{L_{\mathrm{A} 6}}{\mathrm{~A}_{\mathrm{A} 6}}$ \\
\hline A2 & $\left(\begin{array}{lll}1 & 1 & 1\end{array}\right)$ & 0 \\
\hline A3 & $\left(\begin{array}{lll}1 & 1 & 1\end{array}\right)$ & 0 \\
\hline A6 & $\left(\begin{array}{lll}1 & 1 & 1\end{array}\right)$ & 0 \\
\hline \multirow[t]{2}{*}{ B2 } & $\left(\begin{array}{lll}1 & 1 & \overline{3}\end{array}\right)$ & $2 \sqrt{\frac{2}{11}}$ \\
\hline & $\left(\begin{array}{llll}2 & 1 & 1\end{array}\right)$ & 1 \\
\hline \multirow[t]{2}{*}{ B4 } & $\left(\begin{array}{lll}0 & 1 & 1\end{array}\right)$ & $\frac{1}{\sqrt{3}}$ \\
\hline & $\left(\begin{array}{lll}1 & 1 & \overline{1}\end{array}\right)$ & $\frac{2}{3} \sqrt{2}$ \\
\hline \multirow[t]{2}{*}{ B5 } & $\left(\begin{array}{lll}1 & 0 & 0\end{array}\right)$ & $\sqrt{\frac{2}{3}}$ \\
\hline & $\left(\begin{array}{lll}0 & 0 & \overline{1}\end{array}\right)$ & $\sqrt{\frac{2}{3}}$ \\
\hline \multirow[t]{2}{*}{$\mathrm{C} 1$} & $\left(\begin{array}{lll}1 & 0 & \overline{1}\end{array}\right)$ & $\frac{1}{\sqrt{3}}$ \\
\hline & $\left(\begin{array}{llll}1 & 1 & 1\end{array}\right)$ & $\frac{2}{3} \sqrt{2}$ \\
\hline \multirow[t]{2}{*}{$\mathrm{C} 3$} & $(12 \overline{1})$ & 1 \\
\hline & $\left(\begin{array}{lll}1 & 1 & 3\end{array}\right)$ & $2 \sqrt{\frac{2}{11}}$ \\
\hline \multirow[t]{2}{*}{ C5 } & $\left(\begin{array}{lll}0 & 0 & 1\end{array}\right)$ & $\sqrt{\frac{2}{3}}$ \\
\hline & $\left(\begin{array}{lll}0 & \overline{1} & 0\end{array}\right)$ & $\sqrt{\frac{2}{3}}$ \\
\hline \multirow[t]{2}{*}{ D1 } & $\left(\begin{array}{ll}1 & 1\end{array}\right)$ & $\frac{1}{3}$ \\
\hline & $\left(\begin{array}{lll}3 & 1 & 1\end{array}\right)$ & $4 \sqrt{\frac{2}{33}}$ \\
\hline \multirow[t]{2}{*}{ D4 } & 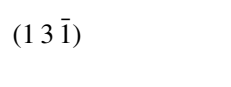 & $4 \sqrt{\frac{2}{33}}$ \\
\hline & $(13 \overline{1})$ & $\frac{1}{3}$ \\
\hline D6 & $\begin{array}{l}\in \text { plane containing } \\
m_{\mathrm{D} 6} \text { and } m_{\mathrm{A} 6}\end{array}$ & $\in(0,1)$ \\
\hline
\end{tabular}

The appendix derives the calculation of the mean free path:

$$
{ }_{n} h_{l}=\frac{\zeta_{l n} L_{l}}{\sqrt{1-\left(m_{l} \cdot N_{l_{\mathrm{p}}}\right)^{2}}},
$$

and by (3.8)

$$
{ }_{n} h_{l}=\frac{\zeta_{l} \lambda_{l n} L_{l_{\mathrm{p}}}^{\mathrm{c}}}{\sqrt{1-\left(\boldsymbol{m}_{l} \cdot \boldsymbol{N}_{l_{\mathrm{p}}}\right)^{2}}} .
$$

Note although the angle between $\boldsymbol{m}_{l}$ and $\boldsymbol{N}_{l_{\mathrm{p}}}$ may change during plastic slip, the mean path length will remain constant with these changes. Assuming elastic stretch deformation to be 
negligible, the mean free path calculations can thus be done in the reference (undeformed) configuration accounting only for changes in the length ${ }_{n} L_{l}$ and treating $\sqrt{1-\left(\boldsymbol{m}_{l} \cdot \boldsymbol{N}_{l_{\mathrm{p}}}\right)^{2}}$ as a constant.

As was derived by Aubrey and Ortiz (2003) using Orowan's relation, the effect of the dislocation walls shortening the mean free path increases the critical resolved shear stress of the material:

$$
{ }_{n} \tau_{l}^{\mathrm{c}}=\tau_{0}^{\mathrm{c}}+\frac{T}{b_{n} h_{l}}
$$

where $\tau_{0}^{\mathrm{c}}$ is the material-dependent initial critical resolved shear stress, $T$ is the dislocation line tension and $b$ is the Burgers vector. This increase in the critical resolved shear stress with refinement of the subgrain dislocation structures causes both latent and self-hardening in this model.

The added nonlocal energy of the dislocation wall including the increase in $\tau^{\mathrm{c}}$ to both children (i.e. $l$ is the branch in the following equation instead of a leaf) can be inferred from (3.30), (2.32) and (3.12) as

$$
\begin{aligned}
{ }_{n} W_{l}^{\mathrm{DW}} & \equiv \frac{T}{b}\left(\frac{n \gamma_{l^{-}} \lambda_{l^{-}}}{{ }_{n} h_{l^{-}}}+\frac{n \gamma_{l^{+}} \lambda_{l^{+}}}{{ }_{n} h_{l^{+}}}\right) \\
& =\frac{T}{b_{n} L_{l}^{\mathrm{c}}}\left(\frac{n \gamma_{l^{-}}}{\zeta_{l^{-}}} \sqrt{1-\left(\boldsymbol{m}_{l^{-}} \cdot \boldsymbol{N}_{l}\right)^{2}}+\frac{n \gamma_{l^{+}}}{\zeta_{l^{+}}} \sqrt{1-\left(\boldsymbol{m}_{l^{+}} \cdot \boldsymbol{N}_{l}\right)^{2}}\right) .
\end{aligned}
$$

The plastic energy is ${ }_{n} W_{l}^{\mathrm{p}}=\left({ }_{n} \tau_{l}^{\mathrm{c}}\right)\left({ }_{n} \gamma_{l}\right)$, while the dislocation wall energy is ${ }_{n} W_{l}^{\mathrm{DW}}=$ $\frac{T\left({ }_{n} \gamma_{l}\right)}{b\left(h_{n}\right)}$. Thus,

$$
\frac{{ }_{N} W_{l}^{\mathrm{p}}}{{ }_{n} W_{l}^{\mathrm{DW}}}=\frac{b\left({ }_{n} h_{l}\right)\left({ }_{n} \tau_{l}^{\mathrm{c}}\right)}{T} .
$$

Generally for metallic materials the order of $T \approx 10^{-10} \mathrm{~N}, b \approx 10^{-10} \mathrm{~m}$ and $\tau \approx 10^{6} \mathrm{~N} \mathrm{~m}^{-2}$, yielding $W_{l}^{\mathrm{p}} / W_{l}^{\mathrm{BL}} \approx h_{l} \times 10^{6} \mathrm{~m}^{-1}$. Using these estimates, only for laminates larger than approximately $10^{-4} \mathrm{~m}$ may $W^{\mathrm{DW}}$ be treated as a perturbation, although any laminate this large should find forming finer laminates energetically favorable and eventually invalidate this assumption.

Laminates with coplanar slip. The special case of branched laminates with active coplanar slip systems has the children's slip planes parallel to the dislocation wall predicted to form between the children. In this case, the two children's dislocations move in parallel planes and no dislocation wall forms (Ortiz and Repetto 1999). Note that in this model once the angle of the slip plane to the wall normal is taken into account, $h_{l} \rightarrow \infty$, appropriately giving a dislocation wall energy of zero. If there is no dislocation wall energy, the laminate will be able to form at an infinitesimal width and negate any boundary layer energy as well. This gives a total nonlocal energy of zero, meaning branches with identical slip planes on either side are zero-energy structures. Zero-energy structures are obviously problematic. In reality, the value of $h_{l}$ for two coplanar laminates should be the length of the parent structure containing them.

There is a second problem with coplanar laminates. If no dislocation walls form in a coplanar laminate, then there is nothing to prevent the dislocations of future new branching slip systems inside the coplanar laminate from gliding over the entire length of both the coplanar laminates containing the new slip system and its sibling, and thus, forming at the length scale of the coplanar laminate's parent. As this change in length scale is not accounted for in a single slip theory, the children of laminates with coplanar siblings would form at lower length 


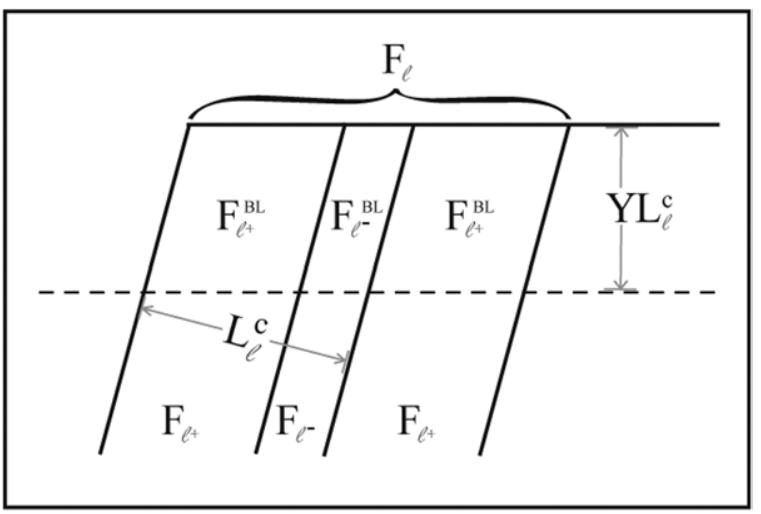

Figure 7. Detailed figure showing boundary layer variables.

scales and thus harden very much. The two problems caused by the lack of dislocation walls in coplanar laminates is the reason for the requirement of a coplanar slip approach and was already discussed in section 2.3.4.

Although these coplanar systems should not only self-harden but harden each other (Franciosi and Zaoui 1982), the results of Franciosi (1985) and the analysis of Bronkhorst (1991) and Havner (2005) suggest that coplanar slip systems should exhibit much less latent hardening than other systems. This work treats coplanar systems as mutually selfhardening (i.e. the slip on two active coplanar slip systems is added and treated as a single slip system which self-hardens). This differs from the traditional multislip theories in which the latent hardening of these systems is often approximated identical to all other systems (Cuitino and Ortiz 1993, Bronkhorst et al 2007).

3.3.2. Boundary layer energy. The boundary layer energy is computed from the elastic deformation that maps the underlying parent material to the two children's deformations. From figure 7 it can be noted that the outside wall of the laminate is at the parent's deformation, ${ }_{n} \boldsymbol{F}_{l}$, while the bulk volume of the children are at their respective deformations, ${ }_{n} \boldsymbol{F}_{l^{ \pm}}$. The boundary layer's state of deformation must change from the parent deformation to the child's deformation. A linear transition is assumed giving the average deformation of the boundary layer as

$$
{ }_{n} \boldsymbol{F}_{l^{ \pm}}^{\mathrm{BL}}=\frac{1}{2}\left({ }_{n} \boldsymbol{F}_{l}+{ }_{n} \boldsymbol{F}_{l^{ \pm}}\right) .
$$

Taking the boundary layer deformation to be elastic yields an energy for the boundary layer,

${ }_{n} W_{l}^{\mathrm{BL}}=\lambda_{l^{+}}\left(W^{\mathrm{e}}\left[\left(\boldsymbol{F}_{l^{+}}^{\mathrm{BL}}\right)\left({ }_{n} \boldsymbol{F}_{l}\right)^{-1}\right]-{ }_{n} W_{l^{+}}\right)+\lambda_{l^{-}}\left(W^{\mathrm{e}}\left[\left(\boldsymbol{F}_{l^{-}}^{\mathrm{BL}}\right)\left({ }_{n} \boldsymbol{F}_{l}\right)^{-1}\right]-{ }_{n} W_{l^{-}}\right)$,

where the terms ${ }_{n} W_{l^{ \pm}}$are subtracted as a correction to the total energy since the energy of the boundary layer volume was assumed to have the child's deformation in previous calculations. Note that the boundary layer energies will remain positive as the energy of the child deformation subtracted involves a low-energy plastic deformation, while the energy of the boundary layer is elastic. Recall that the definition of ${ }_{n} W_{l^{ \pm}}$is the plastic and elastic energy in the individual leaf and does not include the previous plastic deformation in laminate $l$ and other ancestors of the leaf; thus, the value of $W_{l}^{\mathrm{BL}}$ is actually the difference between the energy in the boundary layer volume and the average bulk deformation energy of the branched laminate. It acts as an energy penalty to laminate formation. 


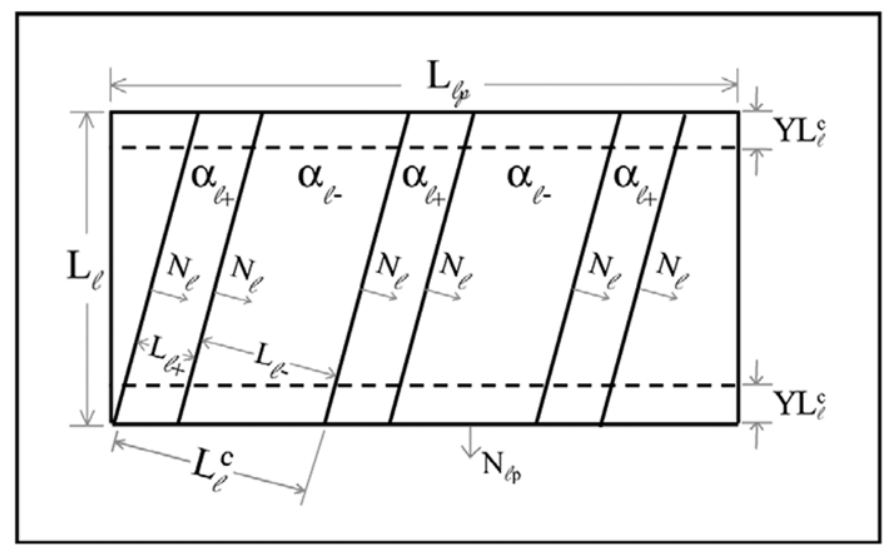

Figure 8. Calculation of the boundary layer volume. Illustration not to scale.

The formulation given here differs from the work of Aubrey and Ortiz (2003) in the correction of the additional elastic deformation to be $\left(\boldsymbol{F}_{l^{+}}^{\mathrm{BL}}\right)\left({ }_{n} \boldsymbol{F}_{l}\right)^{-1}$ rather than $\left(\boldsymbol{F}_{l^{+}}^{\mathrm{BL}}\right)$ only. This change is due to the boundary layer accommodating the deformation from the initial parent material to the child's deformation rather than from an initial undeformed state to the child's deformation.

Note that this is an energy density for the boundary layer, and as such must be added only for the portion of material which is in the boundary layer. The contribution to the total energy density is scaled by the fraction of the boundary volume to the total volume of the simple laminate. In figure 8 , assuming the dislocation wall with normal $N_{l_{\mathrm{p}}}$ is circular, the total volume of one layer of the branched laminate $L_{l}$ is $\pi L_{l} L_{l_{\mathrm{p}}}^{2}$. Note that all other layers will be identical. The corresponding boundary area, taking into account all sides, is $2 \pi L_{l_{\mathrm{p}}}^{2}$, neglecting the small contribution $2 \pi L_{l} L_{l_{\mathrm{p}}}$ justified by $L_{l_{\mathrm{p}}} \gg L_{l}$. Assuming a boundary layer depth of $\Upsilon\left(L_{l}^{\mathrm{c}}\right)$, the total boundary volume is $2 \Upsilon\left(L_{l}^{\mathrm{c}}\right) \pi L_{l_{\mathrm{p}}}^{2}$. Note that the geometric assumption of a circular area is cancelled in the ratio of the volume of boundary layer to total volume, and thus the geometric assumption of circular domain has little influence on this calculation. Using this ratio, the total nonlocal energy density scales as

$$
W_{l}^{\mathrm{NL}}=W_{l}^{\mathrm{DW}}+2 \frac{\Upsilon L_{l}^{\mathrm{c}}}{L_{l}} W_{l}^{\mathrm{BL}},
$$

where $\Upsilon$ is a scaling factor determining the depth the boundary layer penetrates in the laminate in multiples of $L_{l}^{\mathrm{c}}$.

In the work of Ortiz et al (2000) showing boundary layers composed of refining laminate structures, the boundary layer penetration is on the order of $L_{l}^{\mathrm{c}}$. Thus, it can be assumed $\Upsilon$ should be somewhere on the order of 1 . Aubrey and Ortiz (2003) indirectly assign the boundary layer to have a depth of $\frac{1}{2} L_{l}^{\mathrm{c}}$.

As the boundary layer term is scaled by $L_{l}^{\mathrm{c}} / L_{l}$ and it is assumed $L_{l}^{\mathrm{c}} \ll L_{l}$, the boundary layer contribution to the total energy can be assumed small. Due to the minimal contribution, it appears justified to treat it as a perturbation when solving for $\boldsymbol{a}_{l}$.

\subsection{Equilibration updates}

As the macroscopic deformation, ${ }_{n} \boldsymbol{F}$, increases with each time step, it is necessary to update the values of the polarization vectors, ${ }_{n} \boldsymbol{a}_{l}$, in order to keep the stress compatible across the dislocation walls. The values of laminate thickness, ${ }_{n} L_{l}^{\mathrm{c}}$, are also updated as it provides a 
hardening behavior, and mathematically the thickness depends on variables that are being updated, ${ }_{n} W_{l}^{\mathrm{BL}}$ and ${ }_{n} \gamma_{l}$. If the thicknesses were not updated, but rather formed at a single step and held constant afterward, the values of the thicknesses would depend on such things as step size. This is not a desired behavior.

It is desirable to allow multiple rank structures to form within the same time step, e.g. a leaf may branch into two children, then at the same time step those children may further branch into children and so on. The addition of equilibrating the laminate thicknesses for the entire structure described in this section allows multiple branches to form in the same time step. If the thicknesses are not equilibrated as an entire structure, having a laminate with $\gamma=0$ would cause instabilities with $W^{\mathrm{BL}}$ when computing thickness for the children of this laminate. When multiple rank branches form at the same time step, the $\gamma=0$ parents with no plastic deformation themselves essentially become space holders for the children.

It should be noted that a complete optimization of the laminate structure would require that the equilibration be done simultaneously with the formation of new branches. Essentially, when checking whether a new branch forms the structure should be equilibrated, as well as checking every possible combination of other new branches in the tree and equilibrating each combination. Obviously, this problem quickly becomes nontractable. To avoid this, the structure from the previous time step is equilibrated at the beginning of each time step for the new deformation gradient, ${ }_{n} \boldsymbol{F}$, and then each leaf is checked individually to see whether it branches. This process of equilibration and branching is repeated until no new leaves form.

To remove elastic leaves, a minimum limit should be set on the slip strain, $\gamma_{l}$, for a branch to form. Without this restriction leaves may form, which after equilibration deform only elastically. Since the laminates in this theory are based on the formation of subgrain dislocation structures, if a leaf is reacting only elastically, then it should not have been formed. To avoid dependence on the size of the time steps, a small numerical restriction of $\gamma_{l} \geqslant 1 \times 10^{-14}$ is chosen. This restriction is small enough to remove only leaves with energy benefits on the order of computational error. Elastic leaves did not form in any of the simulations presented in this paper.

From this point onward, the subscript denoting step discretization $n$ will be avoided. As the following calculations in this section show the equilibration for a single time step, it is understood that all values have the same $n$.

3.4.1. Compatibility of stress and evolution of the polarization vector. As each new macroscopic deformation step, $\boldsymbol{F}$, is introduced, the equilibration of the stresses across the dislocation walls needs to be updated. Equation (3.18) must be satisfied for each branch simultaneously. The value of the deformation jump $\boldsymbol{a}$ is recomputed for branches in the microstructure using the new global $\boldsymbol{F}$. This was shown and implemented by Ortiz et al (2000). Note that only the leaves in the microstructure are active and free to evolve new values of strain, hence only the leaves obtain new elastic and plastic strain values to accomplish this equilibration.

Noting that the optimization of the deformation jumps, $\boldsymbol{a}_{l}$, and the laminate thicknesses, $\boldsymbol{L}_{l}^{\mathrm{c}}$, are interrelated, an iteration similar to that used in the solution of individual branches is used. The values of $\boldsymbol{a}_{l}$ are solved for using the previous values of $\boldsymbol{L}_{l}^{\mathrm{c}}$, after which the values of $\boldsymbol{L}_{l}^{\mathrm{c}}$ are optimized for the new values of $\boldsymbol{a}_{l}$. This is repeated until the changes in $\boldsymbol{L}_{l}^{\mathrm{c}}$ reach a specified tolerance.

3.4.2. Evolution of laminate thickness. In this theory, the dislocation walls form at an instant, whereas in real materials the dislocation wall will form slowly as more dislocations entangle 
and form dislocation walls. The evolution of thickness over time approximates the formation of the walls over time and refinement of the length scale as new dislocations form between the existing dislocation walls. Further motivation is provided in Hansen (2009).

The minimization of the nonlocal energy with respect to all $L_{l}^{\mathrm{c}}$ is

$$
\min _{\left\{L_{l}^{\mathrm{c}} \mid l=1, \ldots, Z\right\}} \sum_{l^{\prime}=1}^{Z} W_{l^{\prime}}^{\mathrm{NL}},
$$

where $Z$ is the number of laminates in the microstructure. Noting that the only lengths that influence the nonlocal energy of a particular laminate $l$ are $L_{l}^{\mathrm{c}}$ and $L_{l}$ (see (3.23)), the condition for minimization of the total nonlocal energy with respect to a specific $L_{l}^{\mathrm{c}}$ is

$$
\frac{\partial \sum_{l^{\prime}=1}^{Z} W_{l^{\prime}}^{\mathrm{NL}}}{\partial L_{l}^{\mathrm{c}}}=\frac{\partial W_{l^{-}}^{\mathrm{NL}}}{\partial L_{l}^{\mathrm{c}}}+\frac{\partial W_{l^{+}}^{\mathrm{NL}}}{\partial L_{l}^{\mathrm{c}}}+\frac{\partial W_{l}^{\mathrm{NL}}}{\partial L_{l}^{\mathrm{c}}}=0
$$

which yields

$$
L_{l}^{\mathrm{c}}=\sqrt{\left(\frac{L_{l^{+}}^{\mathrm{c}} W_{l^{+}}^{\mathrm{BL}}}{\lambda_{l^{+}}}+\frac{L_{l^{-}}^{\mathrm{c}} W_{l^{-}}^{\mathrm{BL}}}{\lambda_{l^{-}}}+\frac{\delta_{l}}{2 \Upsilon}\right) \frac{\lambda_{l}}{W_{l}^{\mathrm{BL}}} L_{l_{\mathrm{p}}}^{\mathrm{c}}}
$$

where use has been made of equation (3.8): $L_{l}=\lambda_{l} L_{l_{\mathrm{p}}}^{\mathrm{c}}$ and

$$
\delta_{l} \equiv \frac{T}{b}\left(\frac{\gamma_{l^{+}}}{\zeta_{l^{+}}} \sqrt{1-\left(\boldsymbol{m}_{l^{-}} \cdot \boldsymbol{N}_{l}\right)^{2}}+\frac{\gamma_{l^{-}}}{\zeta_{l^{-}}} \sqrt{1-\left(\boldsymbol{m}_{l^{-}} \cdot \boldsymbol{N}_{l}\right)^{2}}\right) .
$$

Note that each $L_{l}$ depends on its parent and children, thus each parent depends on each of its descendant's lengths and each child's length also depends on each of its ancestor's lengths. This infinite loop of dependences is overcome by starting with a known length of the rank 0 laminate (i.e. the grain size), $L_{0}$, which is a required input. As an initial starting condition, set $L_{0}^{\mathrm{c}}=L_{0}$ and $\lambda_{0}=1$, then a direct iteration by decreasing rank of the structure is possible.

To avoid ambiguity in the above calculations, if $l^{ \pm}$is a leaf, $W_{l^{ \pm}}^{\mathrm{BL}}=0$. Note in this case (3.24) is returned.

Hardening. Both traditional mechanisms of hardening, self-hardening and latent hardening are reproduced by the laminate size evolution. As the slip strain within a laminate increases with deformation, the boundary layer energy increases. The simplest approximation for the boundary layer energy density is $W^{\mathrm{BL}}=\frac{1}{2} \mu \gamma^{2}$, where $\mu$ is the shear modulus. Considering (3.24), the laminate size will decrease with increasing strain, $L_{l}^{\mathrm{c}} \propto 1 / \sqrt{\gamma}$. Due to (3.30) this causes a form of self-hardening. When a new slip system is activated, the material must branch, and thus both the new laminate and the existing one will decrease in size, causing a form of latent hardening.

It is also interesting to note that the magnitude of latent hardening is dependent upon the systems that are activated. The changes in magnitude of hardening are caused by the variance in the mean free path of the systems (cf table 2). The results of the mean free path calculations display a similar relationship for coplanar, collinear and other forms of hardening in multiple slip as described by Franciosi and Zaoui (1982) and Havner (2005).

\subsection{Stress jumps on laminate formation}

When a laminate forms there is a discontinuity in the stress state. This is important as any finite element method generally uses iterative solutions to apply traction boundary conditions and to obtain equilibration between elements. This is overcome when necessary by fixing the microstructure during iterations to satisfy a boundary condition. Details of this are discussed in Hansen (2009). 
Table 3. Copper material constants.

\begin{tabular}{lll}
\hline Constant & Value & Reference \\
\hline Elastic constant $\left(C_{11}\right)$ & $168.4 \mathrm{GPa}$ & Simmons and Wang (1971) \\
Elastic constant $\left(C_{12}\right)$ & $121.4 \mathrm{GPa}$ & Simmons and Wang (1971) \\
Elastic constant $\left(C_{44}\right)$ & $75.4 \mathrm{GPa}$ & Simmons and Wang (1971) \\
Critical shear stress $\left(\tau^{\mathrm{c}}\right)$ & $1.0 \mathrm{MPa}$ & Barrett (1952) \\
Burgers vector $(b)$ & $2.56 \times 10^{-10} \mathrm{~m}$ & Kocks et al $(1975)$ \\
Line tension $(T)$ & $18.3 \times 10^{-10} \mathrm{~N}$ & Kocks et al $(1975)$ \\
\hline
\end{tabular}

\section{Results and discussion}

\subsection{Material properties of copper}

A significant feature of the laminate subgrain model is the small number of material constants required, all of which are obtained directly from independent experimental data rather than from numerical fitting. The material constants used for the copper simulations along with references are listed in table 3.

Dislocation line tension, $T$, is approximated using the method given in Kocks et al (1975):

$$
\begin{aligned}
& T_{\text {screw }} \approx \frac{\mu b^{2}}{2}, \\
& \frac{T_{\text {edge }}}{T_{\text {screw }}}=1.76, \\
& T=\sqrt{T_{\text {screw }} T_{\text {edge }}},
\end{aligned}
$$

where $T_{\text {screw }}$ and $T_{\text {edge }}$ are the line tension for a screw and edge dislocation and $\mu$ is the shear modulus given in Kocks et al (1975) as $42.1 \mathrm{GPa}$.

The width of the boundary layer in units of $L^{\mathrm{c}}, \Upsilon$, is set to $1 / 2$ for these simulations. This is the same value as used in Aubrey and Ortiz (2003).

The initial size of the single crystal specimens used in the experiments of Franciosi (1985) and Franciosi and Zaoui (1982) is not known for model validation. As the size directly affects the hardening behavior, the sizes are approximated based upon the stress-strain data. Further details are given in the discussion.

\subsection{Copper single crystal simulations}

Validation of the stress response of copper single crystals can be accomplished by comparison with the experimental data of Franciosi (1985) and Franciosi and Zaoui (1982). Franciosi (1985) states that single crystal copper specimens were tested; each with a different crystal direction aligned to the tensile axis. The crystal directions were [0 0101$],\left[\begin{array}{llll}1 & 0 & 1\end{array}\right],\left[\begin{array}{lll}1 & 0 & 2\end{array}\right]$,

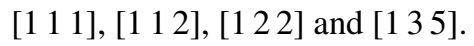

The simulated stress normal to the tensile axis and that measured experimentally for selected orientations are presented. The evolution of the subgrain dislocation structure is also shown. A consistent deformation step of $10^{-3}$ is used for all simulations. All deformations with this model are assumed quasistatic as no rate dependences are incorporated yet. A discussion of the salient structure formed and a comparison of these tests follow. Afterward, the same test conditions are employed to make comparisons of grain size effects against a traditional Hall-Petch effect and to demonstrate the effect of grain shape. 


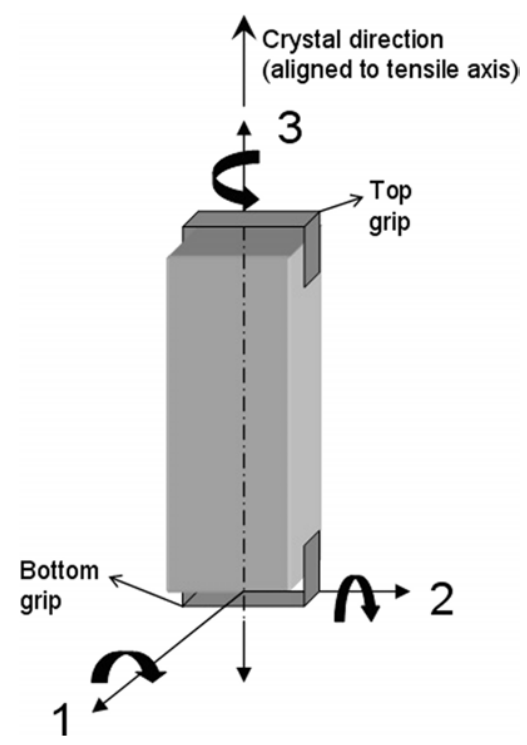

Figure 9. Sample coordinate frame with fixed grip orientation for tensile $\mathrm{Cu}$ specimens.

4.2.1. Boundary conditions. The boundary conditions employed cannot be taken directly from publications. Following the assumption of fixed grips seems to produce appropriate results. Note that for single crystals the choice of boundary conditions is paramount, as the deformation response is far from axisymmetric. In a recent work Shade et al (2009) investigated the effects of boundary conditions on single crystal microcompression. Figure 9 gives the sample coordinate system and visualization for the grips. With the assumption of fixed grips, the boundary conditions used for simulation are given in Hansen (2009):

$$
\begin{aligned}
& \boldsymbol{F}=\left(\begin{array}{ccc}
F_{11} & 0 & 0 \\
0 & F_{22} & 0 \\
0 & 0 & F_{33}
\end{array}\right), \\
& \boldsymbol{P}=\left(\begin{array}{ccc}
0 & P_{12} & P_{13} \\
P_{21} & 0 & P_{23} \\
P_{31} & P_{32} & P_{33}
\end{array}\right),
\end{aligned}
$$

where nonzero values are either an input $\left(F_{33}\right)$ or are to be solved, directly from the material model if a component of $\boldsymbol{P}$, or if an $\boldsymbol{F}$ component, through iteration to get the specified $\boldsymbol{P}$ component as is typical in finite element calculations.

It should be noted that these conditions leave the possibility for significant shear stresses to form. These shear stresses would be supported by the grips. The shear stress components would not have been measured experimentally.

4.2.2. Copper single crystal validation. Figure 10 shows a comparison between simulated and experimental stress-strain curves for copper single crystals. Three different crystal alignments are shown: [0 $\left.\begin{array}{lll}0 & 1\end{array}\right],\left[\begin{array}{lll}1 & 0 & 1\end{array}\right]$ and [ [ 102$]$. The crystal direction is aligned to the tensile axis. The single points are the experimental data from Franciosi (1985); the lines are computer simulations using the laminate hardening model. Note that there are two sets of experimental 


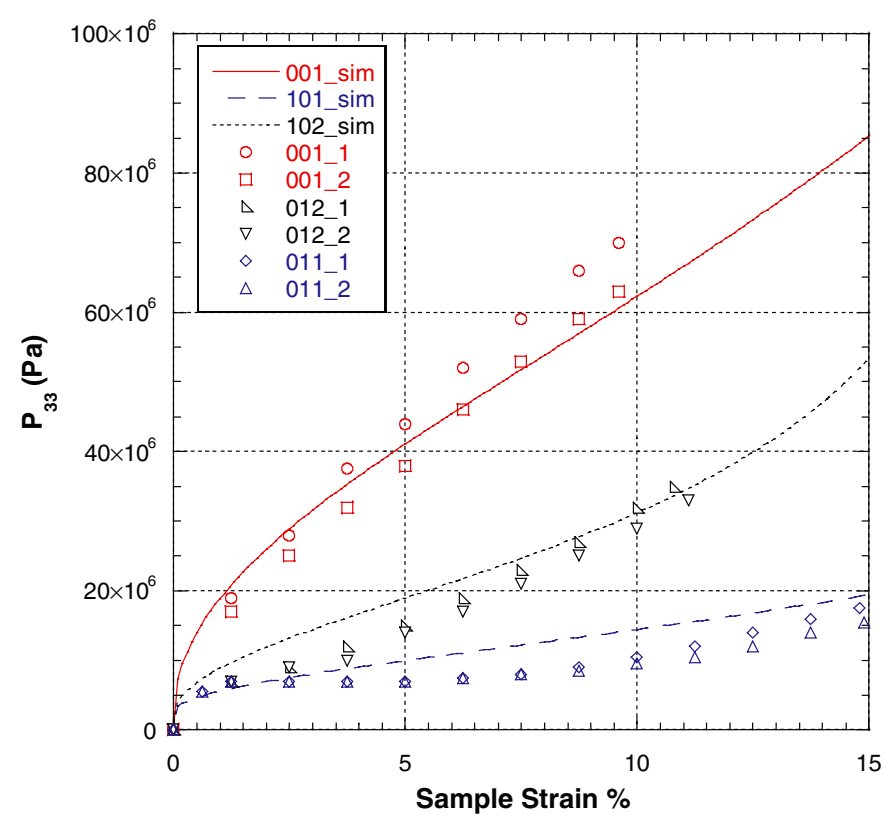

Figure 10. Comparison of experimental and simulation data for a single crystal of copper.

data for each crystal orientation. The simulations provide good validation for the hardening mechanisms of the model for these highly symmetric crystal orientations.

Unfortunately, the size of the samples is not known. Knowing the size and orientation of each sample would define the initial grain size parameter in the simulations. As they are unknown, the sizes are adjusted to resemble the experimental data. The sizes used for the

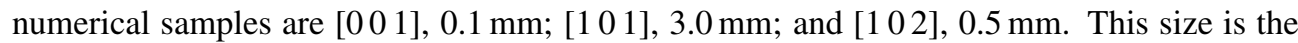
initial length perpendicular to the dislocation wall formed and not the sample diameter, and thus could vary by sample even if the sample shapes are identical. Although this amount of variation on the samples is reasonable for an experimental setup, it is also important to note that the initial grain size parameter and the boundary layer energy are coupled. From (3.24), the length scale of the rank one laminates varies as $\sqrt{L_{\mathrm{o}} /\left(\Upsilon W^{\mathrm{BL}}\right)}$. Hence doubling the boundary layer energy depth or magnitude is equivalent to halving the initial grain size, and so forth. In conclusion, although the initial grain size has been varied in the single crystal simulations, it is equivalent to varying the boundary layer. It would not be unreasonable that different subgrain structures would have varying boundary energy depths, i.e. varying $\Upsilon$. Thus, these two unknowns are coupled. Changes to the number of slip systems activated (for example, by modifications to the assumed boundary conditions) would also affect these results.

The subgrain structure formed during the [ $\left[\begin{array}{lll}1 & 0 & 1\end{array}\right]$ simulation and the active slip systems in the crystallographic coordinates are given in figure 11 . The [1 011$]$ orientation forms a simple laminate involving the B2 and B5 slip systems and the D1 and D6 slip systems. The dislocation wall normal, $N$, is parallel to the tensile axis, $T$, meaning the laminates form perpendicular to the tensile direction. A visualization of this structure in the crystal coordinates is given in figure 12 with color representing the regions in which the two different slip planes are active. The black planes within the colored regions show the orientation of the active slip plane. The tensile axis, $T$, is included for reference. 

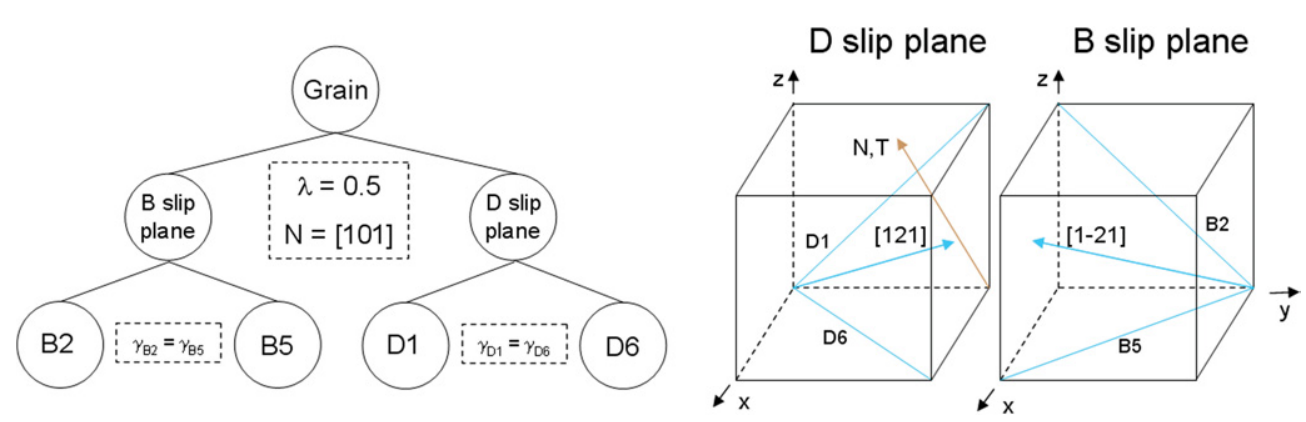

Figure 11. Subgrain structure of [ $\left[\begin{array}{lll}1 & 0 & 1\end{array}\right]$ orientation simulation showing activated slip planes, wall normal, tensile direction and effective slip directions in crystal reference frame

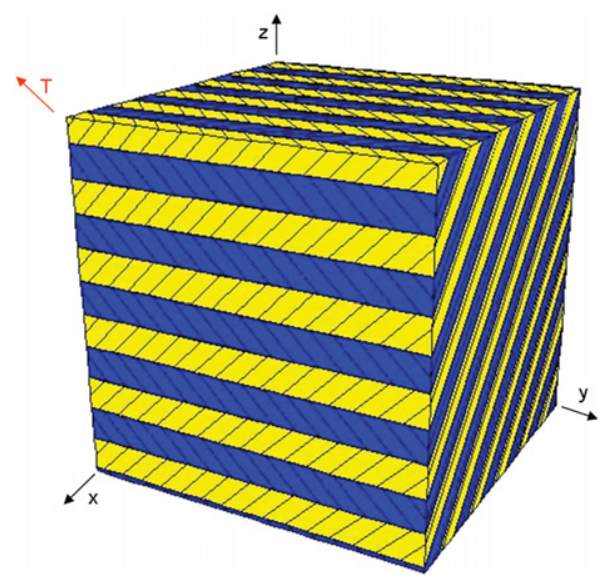

Figure 12. Visualization of [ $\left[\begin{array}{lll}1 & 0 & 1\end{array}\right]$ simulation subgrain microstructure in crystal reference frame ( $\mathrm{T}$ marks the tensile direction).

All four active slip systems have the same strain level as seen in figure 13. The stress state of the sample has no shear stresses that would provide a driving force for the activation of further slip systems. The only nonzero stress in the simulation is the $P_{33}$ component.

Calculation of the Schmidt factor for the [ $\left.\begin{array}{lll}1 & 0 & 1\end{array}\right]$ orientation confirms the four predicted slip systems have the highest Schmidt factor $(\sqrt{6} / 6)$ and should activate first. This is in agreement with the work of Kalidindi and Anand (1993), in which a traditional single crystal model was compared with a [ 10101$]$ copper single crystal deformed in compression. They also predicted the same four slip systems would activate with equal strains. The deformation involves a shortening of the sample 2 direction ( $y$ in the crystallographic coordinate system), while the 3 direction (or tensile axis, $T$, in the crystallographic coordinates) is elongated, and the 1 direction does not change (see figure 9 for the sample coordinate system). This same pattern of deformation is also seen in the work of Kalidindi and Anand (1993) with the experimental copper [ $\left[\begin{array}{lll}1 & 0 & 1\end{array}\right]$-oriented compression sample. The trends are reversed due to the sample being in compression instead of tension (i.e. the 2 direction lengthens, while the 3 direction is compressed and the 1 direction does not change in length). The agreement with deformed shape provides further evidence that the slip systems are correctly predicted. The stress response, activated slip planes and deformed shape are in excellent agreement with 


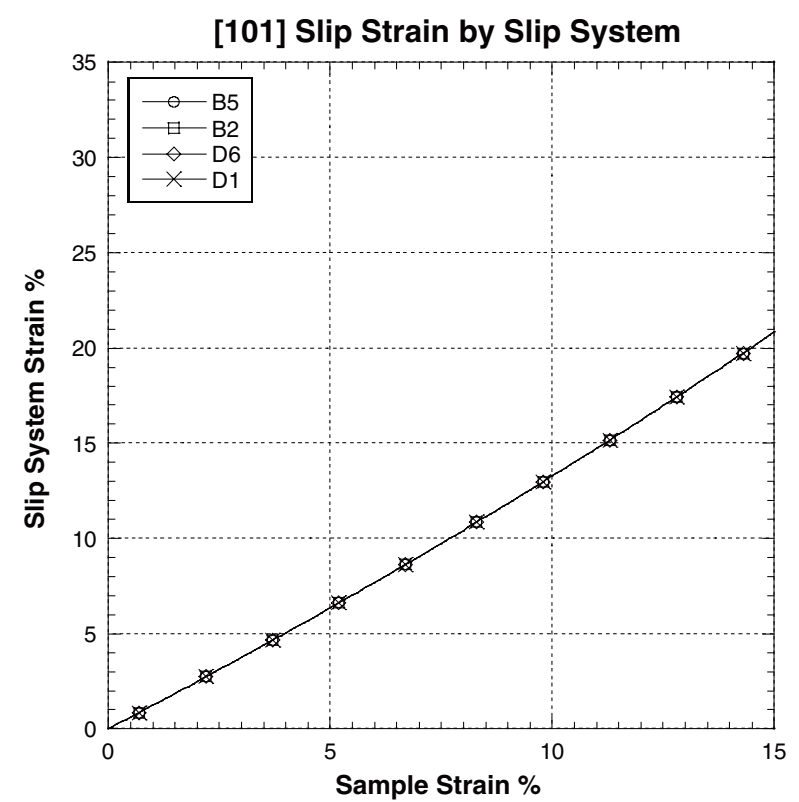

Figure 13. Slip strain on active slip systems for [ $\left[\begin{array}{lll}1 & 0 & 1\end{array}\right]$ simulation.
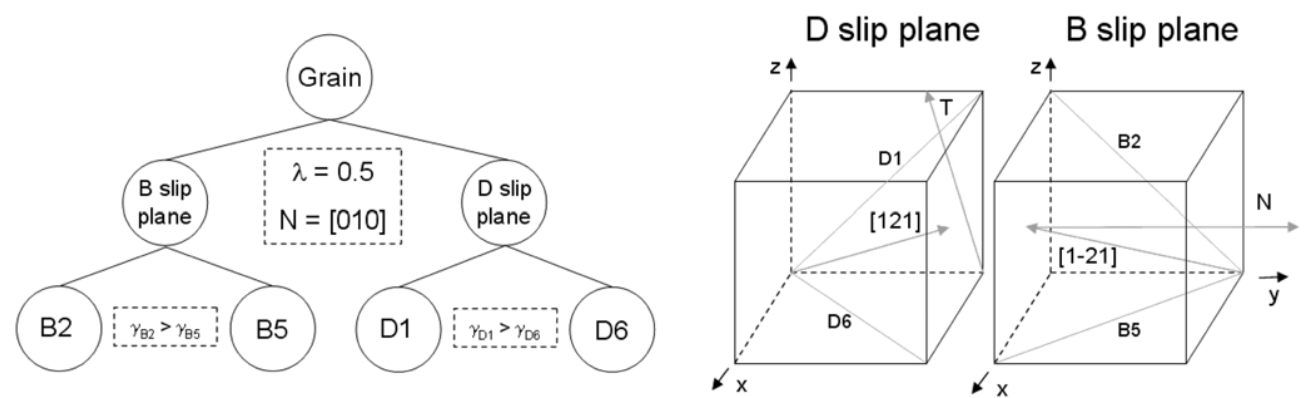

Figure 14. Subgrain structure of [ $\left.1 \begin{array}{lll}1 & 0 & 2\end{array}\right]$ orientation simulation showing activated slip planes, wall normal, tensile direction and effective slip directions in crystal reference frame.

experimental data. It may be useful as a future work to make comparisons with the recent model of Devincre et al (2008).

The subgrain structure formed during the [1 02 ] simulation is given in figure 14. The [1 02 ] orientation forms the same simple laminate involving the B slip plane and the D slip plane as orientation [1 01 ], but a different wall normal. A visualization of the structure is given in figure 15 .

The stress state of the sample has high $P_{13}$ shear stresses that may provide a driving force for the activation of further slip systems, but none were activated at the 15\% strain level of the simulations. Note from the plots of the activated slip strain, figures 16 and 13, that although [ $\left.\begin{array}{lll}1 & 0 & 1\end{array}\right]$ and [ 102$]$ activate the same slip systems, all slip systems do not have the same slip strain in the [1 102$]$ simulation.

The highest Schmidt factors for the [1 02 ] orientation are $\sqrt{6 / 25}$ for systems B2 and D1. The second highest are systems A3, B4, B5, C3, D4 and D6 at $\sqrt{3 / 50}$. This would match 


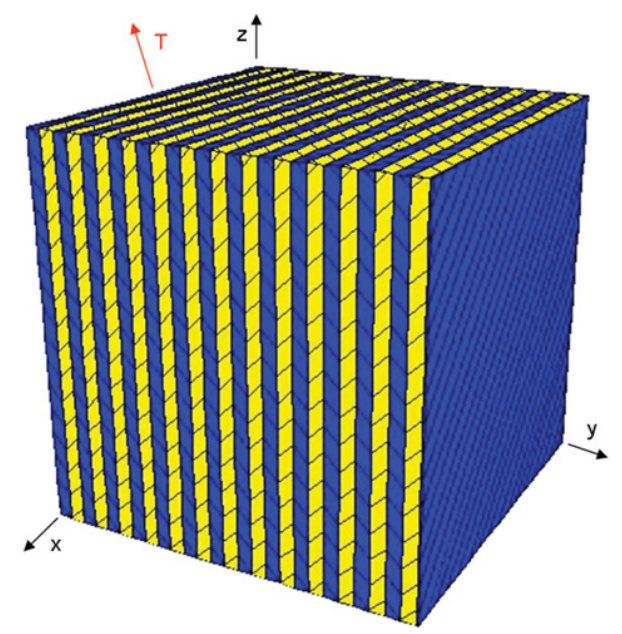

Figure 15. Visualization of [1 02 2] simulation subgrain microstructure in crystal reference frame ( $\mathrm{T}$ marks the tensile direction).

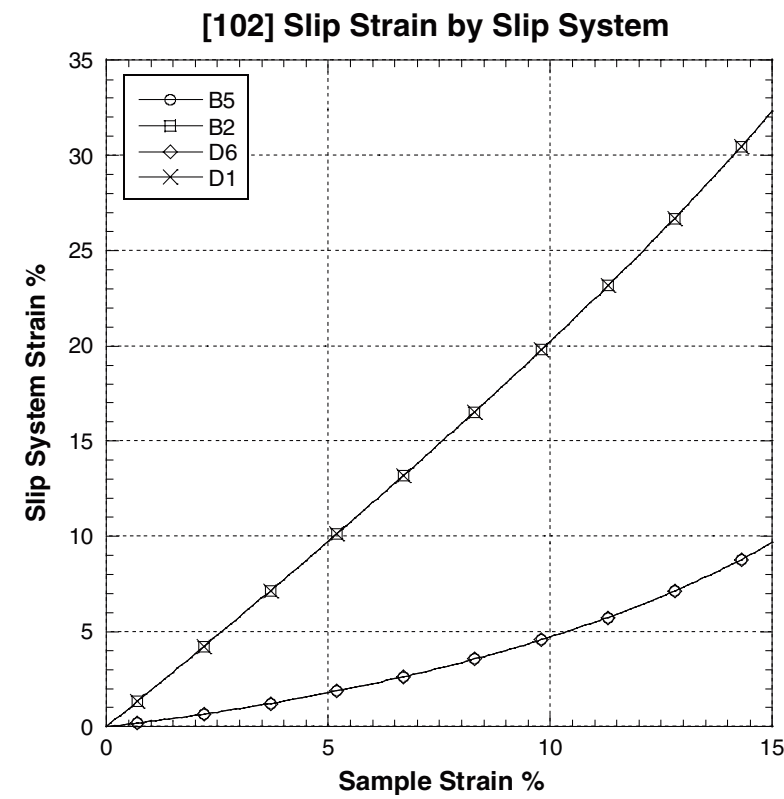

Figure 16. Slip strain on active slip systems for [1 02$]$ simulation.

the prediction from the laminate model that slip systems B2 and D1 have the highest slip strain. The systems B5 and D6 are oriented to relieve the stresses that are not aligned for the simple shear on the B2 and D1 systems. The stress-strain output is in excellent agreement with experimental data.

The [0 001 ] oriented sample activated the slip systems B2, B4, C1 and C3 with identical amounts of slip strain on each system activated as seen in figures 17,18 and 19 . Note that the [0 $\left.0 \begin{array}{ll}0 & 1\end{array}\right]$ simulation activated slip on only one set of two slip planes, namely B and C, but there is an equivalent second set of slip planes, $\mathrm{A}$ and $\mathrm{D}$. The fact that only one set of slip planes is 

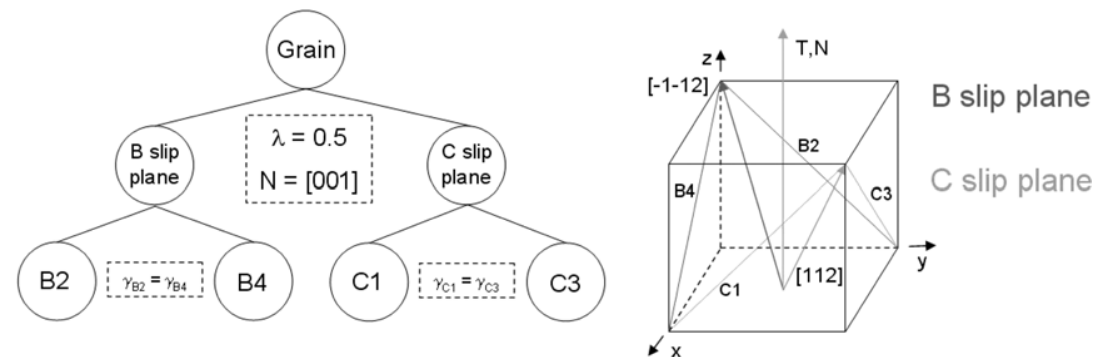

Figure 17. Subgrain structure of [0 01 1] orientation simulation showing activated slip planes, wall normal, tensile direction and effective slip directions in crystal reference frame.

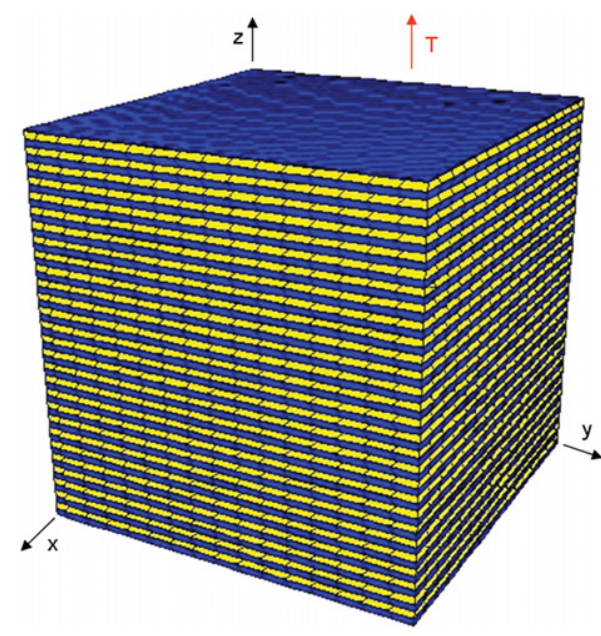

Figure 18. Visualization of [ [ 001 1] simulation subgrain microstructure in crystal reference frame (T marks the tensile direction).

activated means that activating all four slip planes is a higher energy configuration. The [0 01 1] orientation exhibits no shear stresses, as the [ $\left.\begin{array}{lll}1 & 0 & 1\end{array}\right]$ sample; the only nonzero stress component is $P_{33}$.

The highest Schmidt factor for the [ $\left[\begin{array}{lll}0 & 0 & 1\end{array}\right]$ orientation is $\sqrt{6} / 6$ on systems A2, A3, B2, B4, C1, C3, D1 and D4. The traditional model of Cuitino and Ortiz (1993) predicts that all eight systems would activate to the same slip strain. If the deformed shape of the sample were available, whether all eight slip systems were activated could be determined by the final shape of the sample. If the final deformed shape is axisymmetric, then all eight slip systems were likely activated as predicted by the Schmidt factor; but if it forms an asymmetric shape, then likely only two slip planes (with four slip systems) activated.

It should also be stated that the rotation of the [lllll 001 sample about the 3 axis is substantially influenced by the boundary conditions. To activate the minimum energy deformation patterns displayed, it was necessary to run the sample with the [1 000$]$ crystal direction parallel to the $\left[\begin{array}{lll}1 & 1 & 0\end{array}\right]$ sample direction. This is evidence again of the importance of boundary conditions. The small sample size for the [ $\left[\begin{array}{lll}0 & 0 & 1\end{array}\right]$ simulation may be caused by unknown boundary conditions in the experiments which are not being matched. As the experimental clamping mechanism and the exact orientation of the [0 0101$]$ sample used are unknown, it is impossible to investigate this without further experiments. 


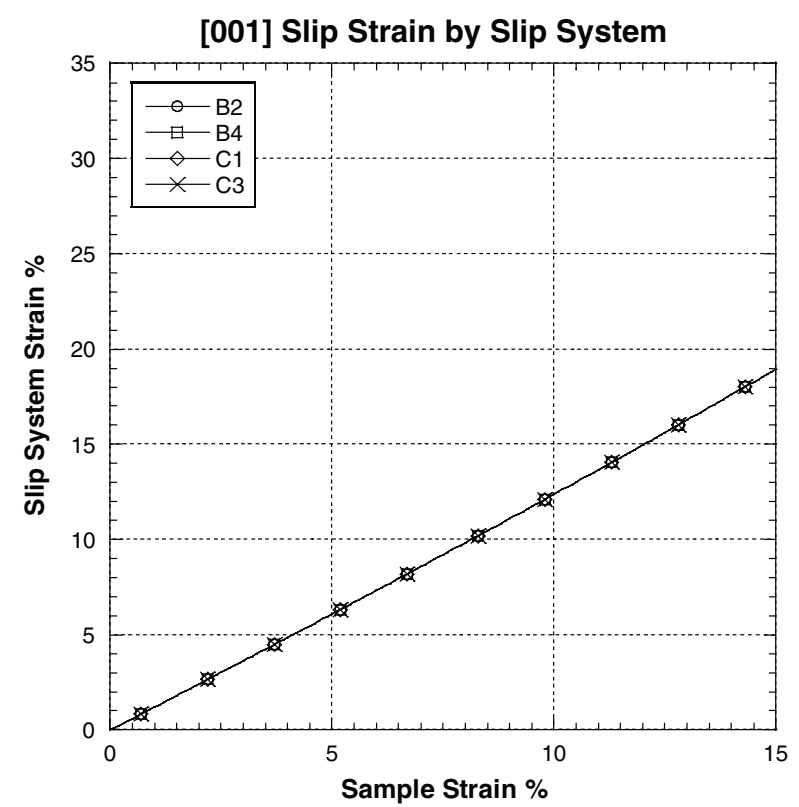

Figure 19. Slip strain on active slip systems for [0 01 1] simulation.

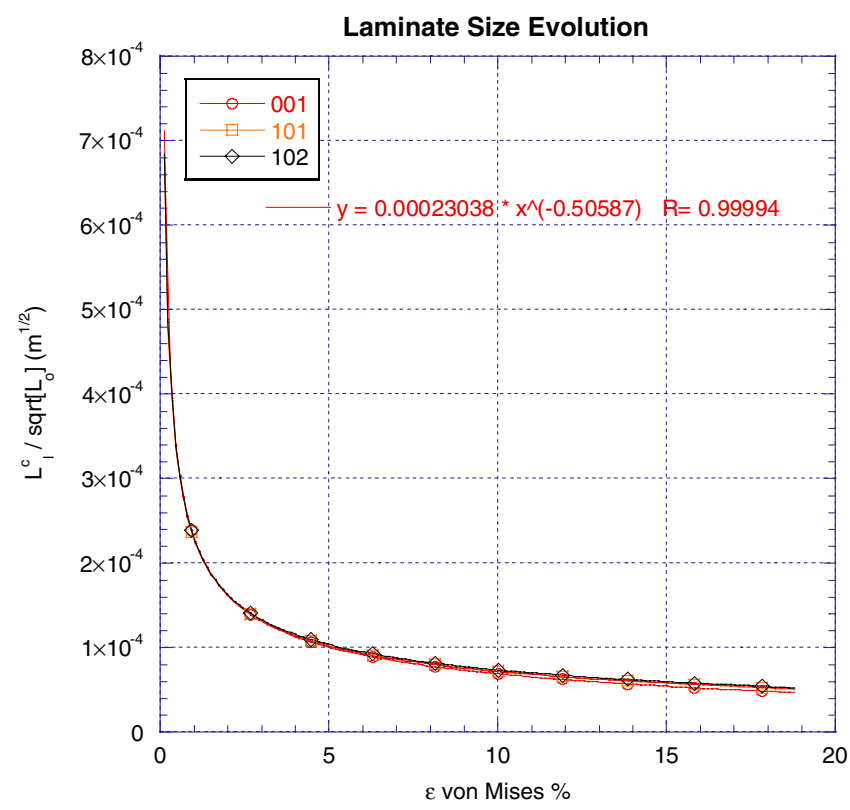

Figure 20. Evolution of laminate thickness scaled by the square root of grain size, $\sqrt{L_{0}}$, against von Mises strain for the three copper simulations: [1 $\left.\begin{array}{lll}1 & 0 & 1\end{array}\right],\left[\begin{array}{lll}1 & 0 & 2\end{array}\right]$ and [ $\left[\begin{array}{lll}0 & 0 & 1\end{array}\right]$.

Figure 20 is a plot of the change in the laminate thickness with deformation. It is scaled by the square root of the grain size to remove the effects of grain size on laminate size (cf (3.38)). Note that it confirms that the laminates refine with increased slip strain causing parabolic hardening. With the effects of grain size removed, the laminates of all orientations evolve 


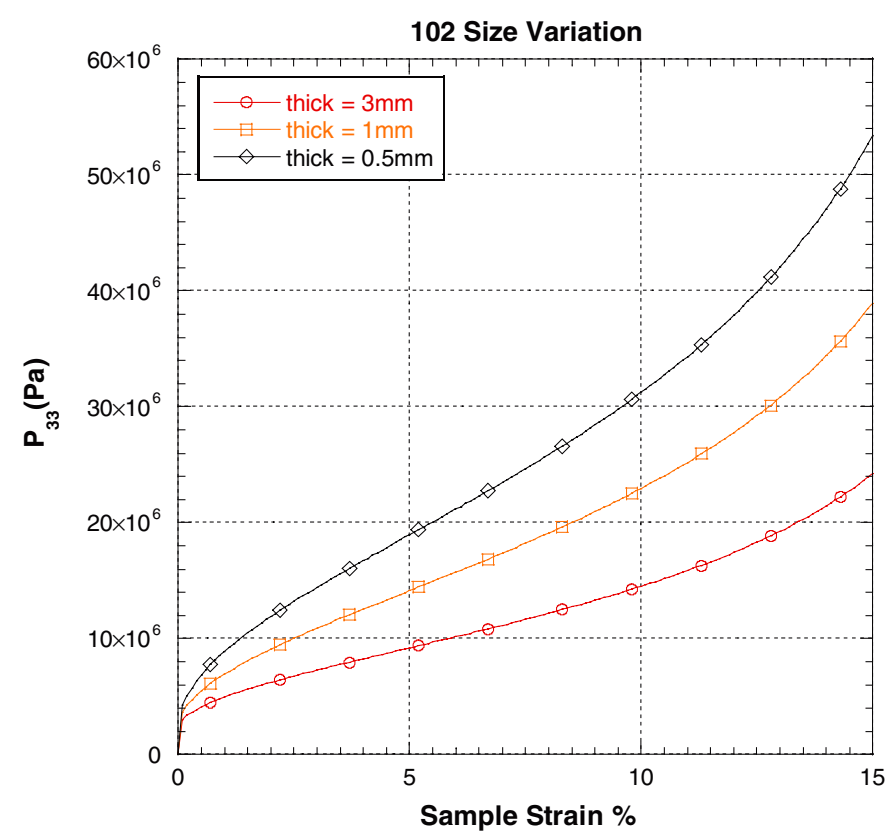

Figure 21. Grain size variation for [ $\left[\begin{array}{lll}1 & 0 & 2\end{array}\right]$ direction copper single crystals.

uniformly with increasing strain. The end result can be fit by $L^{\mathrm{c}} / \sqrt{L_{0}}=0.00023 \epsilon_{\mathrm{vM}}^{-\frac{1}{2}}$, as seen in the figure. This relationship can be derived directly from the equation for $L_{l}^{\mathrm{c}},(3.38)$, and assuming $W_{l}^{\mathrm{BL}} \approx \frac{1}{2} \mu \delta_{l}^{2}$ where $\mu$ is the shear modulus. Note that $\delta$ is a measure of the plastic strain and since the children do not exist, their boundary layer energy is zero. This yields the approximation that $L^{\mathrm{c}} / \sqrt{L_{0}} \propto \delta^{-\frac{1}{2}}$.

Hughes et al (1997) measured in aluminum that the average distance between IDBs increases as $\epsilon_{\mathrm{vM}}^{-\frac{1}{2}}$ and the average distance between GNBs increases as $\epsilon_{\mathrm{vM}}^{-\frac{2}{3}}$. Generally, the laminate model fits GNBs. The difference may be due to uncertainties in the boundary layer energy formulation. Changing the form of the boundary layer energy, (3.34), would produce a different exponent. This is an area that requires further studies.

The von Mises strain measure was used in this section as it was used by Hughes et al for cold rolled samples. The conversion is

$$
\epsilon_{\mathrm{vM}}=-\frac{2}{\sqrt{3}} \ln \left(2-F_{33}\right) .
$$

\subsection{Grain size effects}

Figure 21 displays the effect of varying the initial grain size using the $\left[\begin{array}{ll}1 & 0\end{array}\right]$ ] orientation. The stress increases at a given strain as the initial grain size decreases.

The increase in critical resolved shear stress due to the formation of dislocation walls varies inversely with the square root of the grain size (i.e. $\Delta \tau_{l}^{\mathrm{c}} \propto \frac{1}{\sqrt{L_{0}}}$ ). This is seen by substituting the laminate length, (3.24), into the equation for the mean free path, (3.29), and then considering (3.30). This promises the Hall-Petch effect (Hall 1951, Petch 1953): $\sigma^{y}=\sigma_{\mathrm{o}}+\frac{K}{d^{0.5}}$ where $\sigma^{y}$ is the yield stress, $d$ is the initial grain size and $\sigma_{\mathrm{o}}$ is the initial yield stress parameter. The Hall-Petch effect may be checked directly on the single crystal simulations. This work 


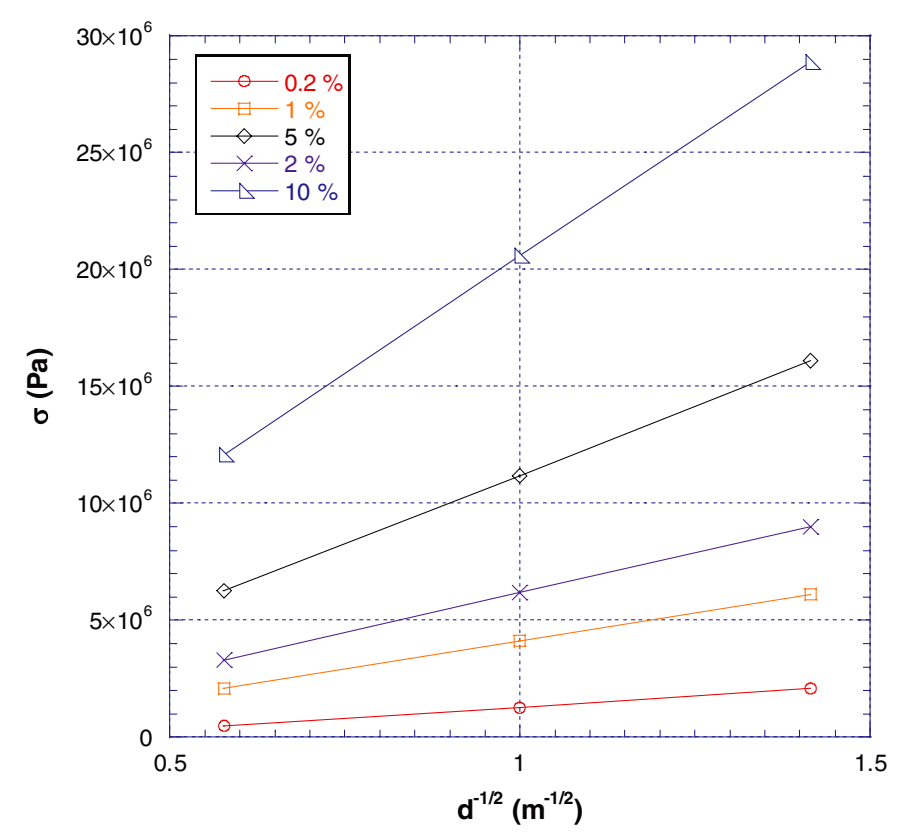

Figure 22. Graph of yield strength at various off-strains versus $\frac{1}{\sqrt{L_{0}}}$ for the $\left[\begin{array}{ll}1 & 0\end{array}\right]$ oriented copper single crystal displaying the Hall-Petch effect.

develops the Hall-Petch effect by modeling of dislocation cell walls. For a review of how a developing dislocation structure yields the Hall-Petch effects see Hansen (2004).

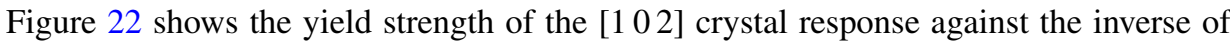
the square root of the initial grain size computed at various offset strains to the linear elastic deformation. The Hall-Petch relationship holds as the plots are linear. This relationship holds as long as a single layer of laminate forms, as was the case in all experiments examined here. It should also hold for higher order laminate structures except during the time steps in which the new laminates form.

Uchic et al (2004) and Greer et al (2005) have recently worked on the deformation response of micrometer sized columns of single crystals. This work displayed that single crystals exhibit a Hall-Petch relationship, despite Hall-Petch having been found for polycrystals. The theory presented here shows the Hall-Petch effect due to the formation of a dislocation subgrain structure with a grain size dependence, hence it provides a possible explanation for the Hall-Petch effect for both single crystals and polycrystals. Both Uchic et al (2004) and Greer et al (2005) also predicted a transition as the sample size decreased to a 'breakaway flow' behavior. This theory would predict that transition as the length scale at which the deformation process no longer favors the formation of dislocation structures, but instead activation of a single slip system. The Uchic et al experimental data are on a $\mathrm{Ni}_{3} \mathrm{Al}-\mathrm{Ta}$ alloy and the Greer et al data are on gold, so no direct comparison is possible for copper considered here. Thus, the theory presented here should follow the same trends discovered on the micrometer-sized single crystals.

\subsection{Grain shape effects}

All simulations to this point have assumed spherical grains. The grain shape will also affect the deformation response as it changes the ratio of boundary layer to total volume and the 
initial length parameter will depend on the wall normal of the first laminate formed. The effect of grain shape is explored by assuming three different grain shapes; all with the [ $\left.\begin{array}{lll}1 & 0 & 1\end{array}\right]$ crystal axis aligned to the tensile axis. The grain shapes simulated are one sphere and two ellipsoids with the major axis, $a$, twice that of the other two axes (prolate spheroids). One ellipsoidal grain has the major axis aligned to the [1 011$]$ crystal axis (and thus aligned to the tensile axis); the other has the major axis aligned to the [0 $\left.\begin{array}{lll}1 & 0\end{array}\right]$ crystal direction (perpendicular to the tensile axis). To get an accurate comparison, the ellipsoidal grains' volumes are set equal to the spherical grain volume. This yields $a=\sqrt[3]{4} L_{0}$ where $L_{0}$ is the diameter of the spherical grain. The simulated spherical grain has a diameter of $3 \mathrm{~mm}$, yielding $a=4.76 \mathrm{~mm}$ for the ellipsoidal grains. Due to increased surface area the elliptical grains have $5 \%$ more boundary layer volume. This condition is arrived at by taking $\Upsilon \approx 1$ and assuming $L^{\mathrm{c}} \ll L_{\mathrm{o}}$, then comparing the boundary layer volume (volume penetrating $\Upsilon L^{\mathrm{c}}$ inside surface) of a sphere and an equal area ellipse with one axis twice the other axes. The boundary layer increase is modeled by setting the parameter $\Upsilon$ to 0.525 for the elliptical grain simulations.

It was assumed that each grain forms the same laminate substructure as was seen for the initial spherical [ $\left.\begin{array}{lll}1 & 0 & 1\end{array}\right]$ simulation (cf figure 11). Since the elliptical grain aligned to the [ 10101$]$ tensile axis forms with dislocation wall normals parallel to the longer ellipse axis, $a$, it has an initial length of $a$ in which to form laminates. The elliptical grain aligned to the [0 10$]$ direction for the same reasons has an initial length of $a / 2$. Thus, the elliptical grain aligned

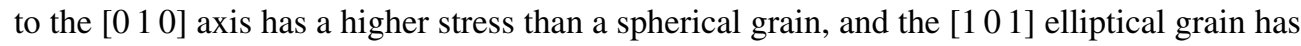
a lower stress than a spherical grain.

The results of these simulations are shown in figure 23. As this grain shape effect is due to the formation of subgrain structures inside the single crystal rather than compatibility constraints within a polycrystalline sample, comparison would need to be made with similar-shaped single crystal tests. As of now, no information is apparent for comparisons.

\section{Conclusions}

A reconfiguration of the laminate model for subgrain dislocation structures has been presented. The model has been modified to include calculation of the mean free path of dislocations incorporating the geometry of the slip plane and the dislocation walls, evolution of the subgrain dislocation laminate length scale over time to capture plastic slip plane hardening, an adjusted boundary layer energy and wall normal implementation, direct minimization of the nonlocal energy terms and resilience to discontinuities in the stress and material tangent. A method for treating the unique case of coplanar slip laminate structures as a single slip system was also developed to get an appropriate hardening behavior for single crystals.

The addition of dislocation mean free path adjusted for the angle of the slip plane within the microstructure provides for a first approximation of shape effects from interactions within the subgrain laminate structure. The inclusion of dislocation mean free path calculations drastically affects the selection of activated slip systems and the hardening behavior of slip systems.

The evolution of the width between dislocation wall subgrain structures provides hardening mechanisms akin to those of self- and latent hardening. The dimensions of the subgrain structure are determined by minimizing the nonlocal energy. The widths are equilibrated at each deformation step. Self-hardening occurs as the laminate width decreases due to increasing boundary layer energy of the laminate microstructure with increased deformation. 

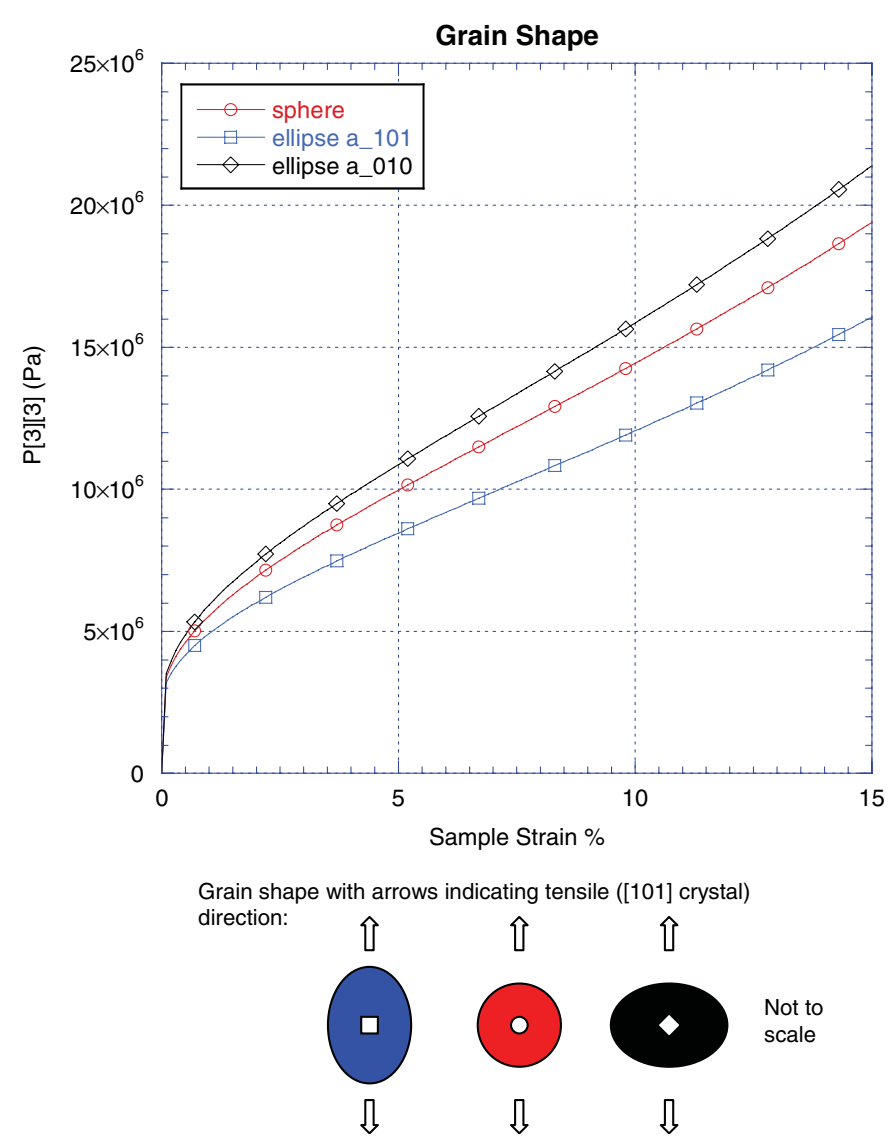

Figure 23. Grain shape effects showing two ellipsoidal grains and one spherical grain for the [ $\left.\begin{array}{llll}1 & 0 & 1\end{array}\right]$ crystal axis.

The crystal bifurcating into separate regions to accommodate activation of new slip systems causes latent hardening to occur. The hardening behavior was validated against experimental tests of copper single crystals. The Hall-Petch effect is returned by the formation of the dislocation subgrain structure. The grain shape effects due to the subgrain structure formation were shown.

The model contains only seven material parameters. All material parameters can be measured directly by independent experiments. Although uncertainties still exist in the effects of the boundary layer energy due to an inherent coupling with the sample size effects, experimental data can be fit very well by varying this energy. Future studies on the boundary layer may provide further refinement of the model.

\section{Acknowledgments}

The authors like to acknowledge the support of the Advanced Simulation and Computing Program (ASC) both at the California Institute of Technology and at Los Alamos National Laboratory in funding this work. 


\section{Appendix A. Derivation of the mean free path length}

The mean free path of dislocations on the slip plane, $h_{l}$, is defined by

$$
h_{l} \equiv \frac{\sum \text { paths }}{\text { \#paths }},
$$

using \#paths to mean the total number of paths. It is assumed that dislocations are generated uniformly at all locations in the plane. Noting that all dislocations (edge and screw) move in the slip plane and that various dislocations mixed between edge and screw may move in any direction to provide the appropriate slip, it is also assumed that the direction of motion of the dislocations is uniformly distributed. The number of paths can be computed

$$
\text { \#paths }=\int_{\Omega} \int_{\alpha=0}^{\alpha=2 \pi} \mathrm{d} \alpha \mathrm{d} \bar{x}=2 \pi A B,
$$

where $\bar{x}$ is the location coordinate on the slip plane and $\Omega$ is the area of the slip plane.

Define $p[\alpha, \bar{x}]$ as the path length of the dislocation starting at $\bar{x}$ and moving at the angle $\alpha$. Thus,

$$
\sum \text { paths }=\int_{\Omega} \int_{\alpha=0}^{\alpha=2 \pi} p[\alpha, \bar{x}] \mathrm{d} \alpha \mathrm{d} \bar{x} .
$$

From figure A1

$$
\begin{aligned}
& \theta_{1}=\arccos \left[\frac{B-x}{\sqrt{(B-x)^{2}+y^{2}}}\right] \\
& \theta_{2}=\arccos \left[\frac{B-x}{\sqrt{(B-x)^{2}+(A-y)^{2}}}\right] \\
& \theta_{3}=\arccos \left[\frac{x}{\sqrt{x^{2}+(A-y)^{2}}}\right] \\
& \theta_{4}=\arccos \left[\frac{x}{\sqrt{x^{2}+y^{2}}}\right] \\
& \alpha_{1}=-\theta_{1} \\
& \alpha_{2}=\theta_{2} \\
& \alpha_{3}=\pi-\theta_{3} \\
& \alpha_{4}=\pi+\theta_{4}
\end{aligned}
$$

This gives

$$
p[\alpha, \bar{x}]= \begin{cases}(B-x) / \cos \alpha & \text { if } \alpha_{1} \leqslant \alpha<\alpha_{2} \\ (A-y) / \sin \alpha & \text { if } \alpha_{2} \leqslant \alpha<\alpha_{3} \\ x / \cos \alpha & \text { if } \alpha_{3} \leqslant \alpha<\alpha_{4} \\ -y / \sin \alpha & \text { if } \alpha_{4} \leqslant \alpha<\alpha_{1}\end{cases}
$$

$B \gg A$ allows for the small angle approximations given in table A1. These approximations are good as long as the point $\bar{x}$ is far from the edges of length $A$, but that area is quite small 


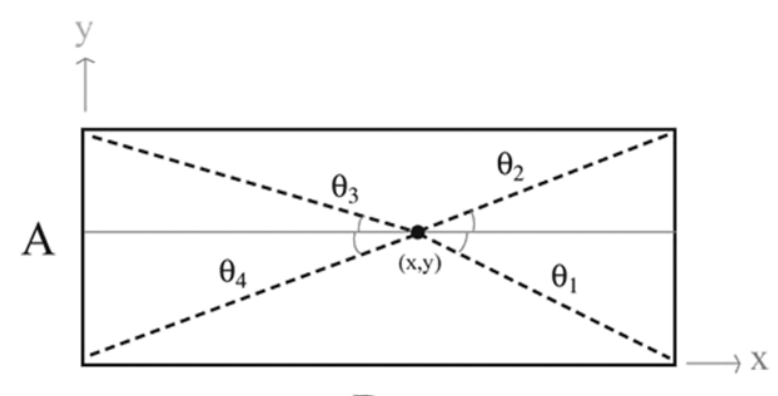

B

Figure A1. Coordinates and angles on the slip plane.

Table A1. Small angle approximations.

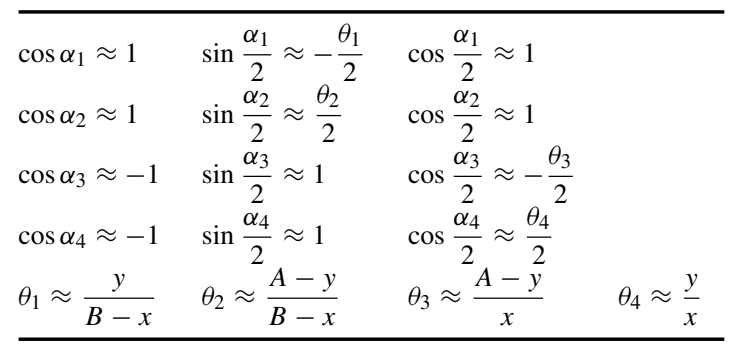

compared with the total area (again due to $B \gg A$ ). Integrating $p$ over $\alpha$ and then applying these approximations yields

$$
\begin{aligned}
\int_{\alpha=0}^{2 \pi} p \mathrm{~d} \alpha \approx & 2 A-y\left(\ln \left[-\frac{y}{4(B-x)}\right]+\ln \left[\frac{y}{x}\right]\right) \\
& +(y-A)\left(\ln \left[\frac{A-y}{4(B-x)}\right]+\ln \left[\frac{y-A}{x}\right]\right) .
\end{aligned}
$$

Then integrating over the domain of the slip plane, and taking only the real part

$$
\int_{\Omega} \int_{\alpha=0}^{2 \pi} p \mathrm{~d} \alpha \mathrm{d} \bar{x}=A^{2} B\left(1+2 \ln \left[\frac{2 B}{A}\right]\right)
$$

yields the mean free path by (A.1)

$$
h_{l}=\frac{A_{l}\left(1+2 \ln \left[\frac{2 B_{l}}{A_{l}}\right]\right)}{2 \pi} .
$$

It is desired to remove the parameter $B$ from the computation of the mean free path as its value is not readily available. The value of $h_{l}$ will be approximated as a linear function of $A_{l}$ using the separation of length scales inherent in laminate structures $(B \gg A)$. The value of $\zeta_{l} \equiv h_{l} / A_{l}$ is investigated. This gives a value for the dislocation mean free path

$$
{ }_{n} h_{l}=\zeta_{l} A_{l}=\frac{\zeta_{l n} L_{l}}{\sqrt{1-\left(\boldsymbol{m}_{l} \cdot \boldsymbol{N}_{l_{\mathrm{p}}}\right)^{2}}},
$$

and by (3.8)

$$
{ }_{n} h_{l}=\frac{\zeta_{l} \lambda_{l n} L_{l_{\mathrm{p}}}^{\mathrm{c}}}{\sqrt{1-\left(\boldsymbol{m}_{l} \cdot \boldsymbol{N}_{l_{\mathrm{p}}}\right)^{2}}} .
$$




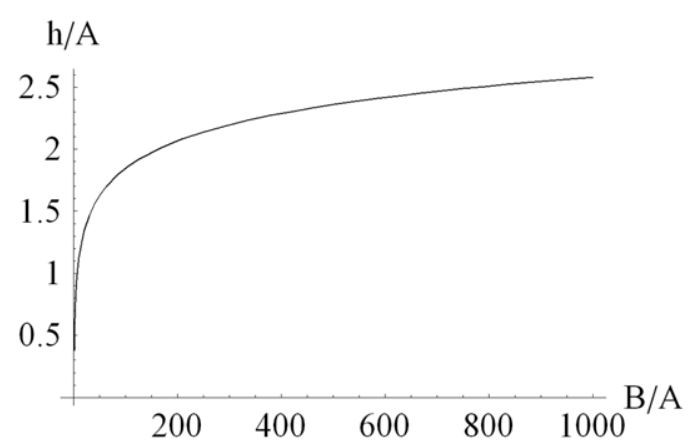

Figure A2. Plot of $\frac{h}{A}$ versus $\frac{B}{A}$.

A plot of $\zeta$ versus $(B / A)$ is given in figure A2, for the range $(B / A) \in[100,1000]$, $\zeta \in[1.846,2.579]$. Due to the small variation of $\zeta$ in a domain of reasonable values, the extra computation is warranted as excessive and the parameter $\zeta$ is approximated as a constant. For this work $\zeta_{l}=2$ is chosen.

\section{References}

Aubrey S and Ortiz M 2003 Proc. R. Soc. Lond. A 459 3131-58

Aubrey S, Fago M and Ortiz M 2003 Comput. Methods Appl. Mech. Eng. 192 2823-43

Barrett C 1952 Structure of Metals (New York: McGraw-Hill)

Bronkhorst C 1991 Plastic deformation and crystallographic texture evolution in face-centered cubic materials $P h D$ Thesis Massachusetts Institute of Technology, appendix $\mathrm{F}$

Bronkhorst C, Hansen B, Cerreta E and Bingert J 2007 J. Mech. Phys. Solids 55 2351-83

Cerreta E 2008 private communication Los Alamos National Laboratory

Conti S and Ortiz M 2005 Arch. Ration. Mech. Anal. 176 103-47

Conti S and Theil F 2005 Arch. Ration. Mech. Anal. 178 125-48

Conti S, Hauret P and Ortiz M 2007 Multiscale Modelling Simul. 6 135-57

Cuitino A and Ortiz M 1993 Modelling Simul. Mater. Sci. Eng. 1 225-63

Devincre B, Hoc T and Kubin L 2008 Science 320 1745-8

Dmitrieva O, Dondl P, Muller S and Raabe D 2009 Acta Mater. 57 3439-49

Franciosi P 1985 Acta Metall. 33 1601-12

Franciosi P and Zaoui A 1982 Acta Metall. 30 1627-37

Greer J, Oliver W and Nix W 2005 Acta Mater. 53 1821-30

Gurses E and Ortiz M 2009 Unpublished manuscript

Hall E 1951 Proc. Phys. Soc. B $64747-53$

Hansen B 2009 Modeling metallic single crystal plastic hardening through the evolution of dislocation subgrain structures $P h D$ Thesis California Institute of Technology (http://resolver.caltech.edu/ CaltechETD:etd_03132009-154225)

Hansen N 2004 Scr. Mater. 51 801-6

Hansen N, Huang X and Hughes D 2001 Mater. Sci. Eng. A 317 3-11

Havner K 2005 Phil. Mag. 85 2861-94

Hughes D and Hansen N 1997 Acta Mater. 45 3871-86

Hughes D, Liu Q, Chrzan D and Hansen N 1997 Acta Mater. 45 105-12

Kalidindi S and Anand L 1993 Metall. Trans. A 24 989-92

Kocks U, Argon A and Ashby M 1975 Progress in Materials Science: Thermodynamics and Kinetics of Slip (New York: Pergamon)

Kohn R 1991 Contin. Mech. Thermodyn. 3 193-236

Lee E 1969 J. Appl. Mech. 36 1-6

Ortiz M and Repetto E 1999 J. Mech. Phys. Solids 47 397-462

Ortiz M and Stainier L 1999 Comput. Methods Appl. Mech. Eng. 171 419-44 
Ortiz M, Repetto E and Stainier L 2000 J. Mech. Phys. Solids 48 2077-114

Petch N 1953 J. Iron Steel Inst. 174 25-28

Rice J 1971 J. Mech. Phys. Solids 19 433-55

Rice J 1975 Continuum mechanics and thermodynamics of plasticity in relation to microscale deformation mechanisms Constitutive Equations in Plasticity ed A Argon (Cambridge, MA: MIT Press)

Rockafellar R 1970 Convex Analysis (Princeton, NJ: Princeton University Press)

Shade P, Wheeler R, Choi Y, Uchic M, Dimiduk D and Fraser H 2009 Acta Mater. 57 4580-7

Simmons G and Wang H 1971 Single Crystal Elastic Constants and Calculated Aggregate Properties: A Handbook (Cambridge, MA: MIT Press)

Steeds J 1966 Proc. R. Soc. Lond. A 292 343-73

Teodosiu C 1982 Elastic Models of Crystal Defects (Berlin: Springer)

Uchic M, Dimiduk D, Florando J and Nix W 2004 Scr. Mater. 51 801-6 